\title{
Étude approfondie sur les adolescents : Exploiter les données pour identifier et atteindre les jeunes les plus vulnérables-Niger 2006
}

Population Council

Follow this and additional works at: https://knowledgecommons.popcouncil.org/departments_sbsr-pgy

Part of the Demography, Population, and Ecology Commons, Family, Life Course, and Society Commons, Inequality and Stratification Commons, and the International Public Health Commons How does access to this work benefit you? Let us know!

\section{Recommended Citation}

"Étude approfondie sur les adolescents : Exploiter les données pour identifier et atteindre les jeunes les plus vulnérables-Niger 2006." New York: Population Council, 2009. 


\section{ÉTUDE APPROFONDIE SUR LES}

\section{ADOLESCENTS : EXPLOITER LES DONNÉES POUR IDENTIFIER ET ATTEINDRE LES JEUNES LES PLUS VULNÉRABLES}

Niger 2006

Données, tableaux, graphiques et cartes élaborés à partir des Enquêtes Démographiques et de Santé 


\section{(2) Population Council}

One Dag Hammarskjold Plaza

New York, New York 10017

Téléphone : 001 212-339-0500

Fax : 001 212-755-6052

E-mail : pubinfo@popcouncil.org

www.popcouncil.org

Population Council est une organisation non gouvernementale internationale, sans but lucratif, qui mène des recherches en sciences biomédicales et sociales et en santé publique. Sa mission est d'améliorer le bien-être et la santé reproductive des générations actuelles et futures ainsi que de contribuer à l'avènement d'un équilibre humain, équitable et durable entre les populations et les ressources.

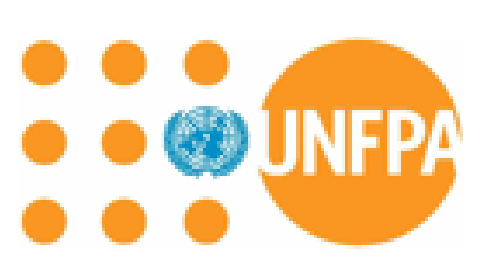

220 E 42nd St \# 21

New York, NY 10017

Téléphone : 001 212-885-2640

www.unfpa.org

L'UNFPA, Fonds des Nations Unies pour la population, est une agence de développement internationale qui œuvre en faveur du droit à la santé et de l'égalité des chances de chacun, femme, homme et enfant. L'UNFPA offre son appui aux pays pour utiliser les données démographiques dans la formulation des politiques et des programmes visant à réduire la pauvreté et pour faire en sorte que chaque grossesse soit désirée, que chaque accouchement soit sans danger, que chacun soit protégé du VIH/SIDA et que toutes les filles et toutes les femmes soient traitées avec dignité et respect.

Toute partie de ce rapport peut être reproduite ou adaptée si besoin est pour être utilisée au niveau local sans l'autorisation du Population Council ou de l'UNFPA. Cependant, les parties reproduites doivent être distribuées gratuitement ou à prix coûtant (sans bénéfice) et la source des documents doit être indiquée et porter la mention suivante : Etude approfondie sur les adolescents : Exploiter les données pour identifier et atteindre les jeunes les plus vulnérables - Niger 2006 (The Adolescent Experience In-Depth: Using Data to Identify and reach the Most Vulnerable Young People: Niger 2006, New York: Population Council, 2009). Toute utilisation commerciale requiert l'autorisation du Population Council. Le Population Council vous serait reconnaissant de lui adresser une copie de tout document dans lequel ce rapport est repris. 


\section{TABLE DES MATIÈRES}

$\begin{array}{ll}\text { I-Avant-propos } & 4\end{array}$

$\begin{array}{ll}\text { II - Notes techniques } & 7\end{array}$

III - Investir en faveur des adolescents : une stratégie pour la réalisation des Objectifs $\quad 8$ du Millénaire pour le développement

IV - Résumé des données de l'EDS sur les adolescents

$\begin{array}{ll}\text { A - Cartographier la grande diversité des adolescents } & 10\end{array}$

$\begin{array}{ll}\text { Répartition de la population } & 11\end{array}$

$\begin{array}{ll}\text { Présence des parents dans le foyer } & 13\end{array}$

$\begin{array}{ll}\text { Survie des parents } & 14\end{array}$

Scolarisation des 10-14 ans $\quad 17$

$\begin{array}{ll}\text { Scolarisation des 15-19 ans } & 18\end{array}$

$\begin{array}{ll}\text { Situation matrimoniale chez les filles } & 20\end{array}$

B - Les transitions importantes qui désavantagent les très jeunes adolescents $\quad 22$

$\begin{array}{ll}\text { Adolescents non scolarisés et ne vivant avec aucun des parents } & 23\end{array}$

Adolescentes non scolarisées et ne vivant avec aucun des parents (carte) 25

$\begin{array}{ll}\text { Scolarisation des 10-17 ans } & 26\end{array}$

$\begin{array}{ll}\text { Enfants en âge d'aller à l'école mais non scolarisés } & 27\end{array}$

Filles en âge d'aller à l'école mais non scolarisées (carte) 28

Analphabétisme chez les filles $\quad 29$

Analphabétisme chez les filles de 15-19 ans (carte) 30

Analphabétisme et mariage avant l'âge de 15 ans 32

Scolarisation en fonction du statut matrimonial et de la maternité 33

C - Contexte de l'activité sexuelle : le mariage précoce, les normes de genre et la santé de la procréation $\quad \mathbf{3 4}$

Age des filles lors du premier mariage ou de la première union $\quad 35$

$\begin{array}{ll}\text { Mariage précoce chez les filles de 20-24 ans (carte) } & 37\end{array}$

Grossesse et maternité $\quad 38$

Activité sexuelle chez les filles de 15-19 ans 40

Initiation sexuelle chez les filles de 20-24 ans 41

Rapports sexuels forcés $\quad 43$

Différence d'âge entre les partenaires lors du premier rapport sexuel $\quad 44$

Différence d'âge entre les partenaires actuels $\quad 45$

Pourcentage des filles qui considèrent que la violence conjugale est
acceptable quelles que soient les circonstances

Pourcentage des filles qui considèrent que la violence conjugale est
acceptable dans certaines circonstances

Dépistage du VIH $\quad 49$

Connaissance des méthodes de prévention du VIH 50

Attitude des filles vis-à-vis de l'utilisation du préservatif masculin 52

Utilisation du préservatif $\quad 52$

Utilisation de contraceptifs $\quad 54$

Santé prénatale $\quad 55$

Assistance lors de l'accouchement $\quad 56$

V-Quelques remarques pour conclure $\quad 58$ 


\section{$\underline{\text { I - Avant-propos }}$}

Le but de la série Fact Book, qui se base essentiellement sur les données des Enquêtes Démographiques et de Santé (EDS), est de fournir à tous les décideurs (au sein des gouvernements, organisations non gouvernementales et groupes de plaidoyer) des données sur la situation des adolescent(e)s et jeunes femmes. La tranche d'âge concernée est celle des 10-24 ans. Les données sont présentées sous forme de graphiques, de tableaux et de cartes (dans la mesure du possible), soit de nombreux formats pour rendre l'information accessible à différents publics.

La section II présente de courtes notes techniques sur chaque pays, en indiquant les sources des données et les tailles des échantillons ainsi que tout avertissement utile pour l'utilisation des données (comme un échantillon trop faible, des problèmes autour de la base d'échantillonnage ou le degré de représentativité des analyses).

La section III apporte une vue d'ensemble des Objectifs du Millénaire pour le développement (OMD) et servira à toute personne désireuse de conjuguer investissements adéquats dans la jeunesse et réalisation des OMD, en particulier dans le cadre des Stratégies de réduction de la pauvreté (SRP) et des agendas de santé sexuelle et de la procréation des jeunes.

La section IV résume certaines données clés des adolescents de 10-24 ans et présente les tableaux, graphiques et cartes en trois grands thèmes destinés à guider les lecteurs dans l'étude : de la diversité des adolescents, des transitions importantes survenant au début de l'adolescence et de la vulnérabilité des adolescents à la puberté, et enfin des circonstances de l'activité sexuelle, de la grossesse et des naissances.

La section IV-A donne une vue d'ensemble des informations concernant la diversité des adolescents de 10-24 ans habitant dans le pays, et met en évidence les différences d'âge, de sexe, de modes de vie, de scolarisation et de situation matrimoniale, et de milieu de résidence actuel (urbain ou rural). Le but de cette section est d'identifier les grandes sous-catégories potentiellement vulnérables d'adolescents et de jeunes qui ont besoin d'une attention particulière. Tant les Stratégies de réduction de la pauvreté que les politiques de jeunesse ont pour objectifs de fournir des systèmes de protection sociale et de donner une seconde chance aux personnes désavantagées. Ces désavantages peuvent être la conséquence de situations sociales (par exemple, avoir fait l'objet d'un mariage précoce ou avoir quitté le domicile familial), de manque de compétences (par exemple : avoir un faible niveau d'études) ou d'un manque d'accès aux ressources matérielles.

La section IV-B met en relief la tranche des 10-14 ans, de manière à identifier les moments de la vie où ils entament une transition vers des phases potentiellement à risque car de nombreux pays ont négligé cette tranche d'âge en dehors des simples droits à la scolarité. La plupart des pays ont pris des engagements pour faire entrer les jeunes à l'école primaire et les faire terminer ce cycle d'études, et s'attendent donc à ce que les adolescents de 10-14 ans soient effectivement scolarisés. Les décideurs politiques présument souvent que les jeunes de 10-14 ans sont sous la protection efficace d'un adulte (idéalement leurs parents). Beaucoup de politiques supposent que ces jeunes constituent une catégorie de population relativement stable, bénéficiant du soutien parental ; c'est pourquoi beaucoup de programmes cherchent à impliquer les 
parents. Or, ces derniers peuvent ne pas être présents dans la vie de ces jeunes. Cette section vise à mettre en lumière les populations de jeunes adolescents exclus, qui ne bénéficient pas nécessairement de toutes les ressources sociales présupposées et qui n'opèrent pas la plus saine des transitions vers l'âge adulte. Ces populations comprennent :

-les personnes qui sont mariées très tôt ;

-les personnes non scolarisées ;

-quand les données le permettent, les personnes vivant sans un ou sans les deux parents

Les jeunes qui vivent sans l'un de leurs parents ou sans les deux sont souvent des orphelins et ont plus de risques de participer à des activités illégales et de faire l'objet d'un mariage précoce. Certains sont parfois déjà mariés et vivent donc avec leur conjoint ou leur belle-famille.

L'arrivée de la puberté amène des changements physiques considérables ainsi que des vulnérabilités chez les garçons et surtout chez les filles. Chez celles-ci, la puberté démarre en moyenne deux ans plus tôt que chez les garçons. Cette différence, associée à des normes de genre très contraignantes et à des ressources limitées, fait que les jeunes filles ne disposent souvent que de leur corps comme ressource essentielle fiable. Cette ressource est susceptible d'être exploitée pour un travail dangereux ou des relations sexuelles non souhaitées, non protégées et précédant la majorité, ou encore d'entraîner un mariage préventif, contraire aux droits et à la volonté de la jeune fille, dans l'optique que celle-ci ait des enfants le plus tôt possible.

La section IV-C met en évidence les circonstances des relations sexuelles, au sein du mariage ou en dehors, et fournit les taux de mariage et de grossesse, ainsi que des données en rapport avec les normes de genre qui encadrent souvent le début et la durée des relations sexuelles et maritales. En outre, des données sur la connaissance et le dépistage du VIH, l'usage de contraceptifs, les soins prénataux et l'aide à l'accouchement sont indiquées chaque fois que possible.

Il n'y a pas de données idéales. Toutefois, l'information présentée est d'une très grande qualité et est largement représentative (voir la section II pour une description de la représentativité des données). Les tableaux, graphiques et cartes ont pour but d'informer, mais aussi de susciter des questions et d'encourager un engagement et une action au niveau local. Ils constituent une base pour l'élaboration d'une seconde génération de programmes pour les adolescents et les jeunes, plus clairement centrée sur des sous-catégories larges et négligées de population à des moments charnières. Comme les politiques actuelles de jeunesse n'ont souvent pas dirigé leurs ressources vers les sous-catégories d'adolescents vulnérables et difficiles d'accès, les fruits de ces politiques ont bénéficié aux sous-catégories les mieux loties (généralement des populations urbaines, plus âgées, masculines, non mariées et scolarisées). Dans la plupart des cas, les populations d'adolescents qui vivent en milieu rural, qui sont des filles, plus jeunes, non scolarisées et mariées sont négligées.

Cette documentation vise à renseigner les débats d'acteurs locaux, les campagnes de plaidoyer et le travail de renforcement des compétences. Le texte qui introduit chaque section et sous-section encadre les données de manière adéquate pour les groupes de plaidoyer qui cherchent à sensibiliser aux besoins des personnes jeunes et vulnérables 
dans leurs pays de prédilection. Le texte et les données sont destinés à aider les analystes politiques comme les acteurs du plaidoyer à :

-établir des conclusions

-prendre des décisions

-quand les données sont vagues ou manquantes, inciter à trouver des clarifications par une politique ciblée et une recherche à destination des programmes.

Ce projet va se poursuivre : au fur et à mesure que des données en provenance d'autres pays et des données plus récentes issues des EDS ou d'autres enquêtes nationales (comme les MICS, PAPFAM ou le LSMS) seront disponibles, de nouveaux guides statistiques seront élaborés. De plus, d'autres tableaux ou indicateurs susceptibles d'intérêt seront rajoutés à mesure qu'ils sont identifiés. À tout moment vos remarques sont les bienvenues.

Enfin, le Population Council et l'UNFPA voudraient remercier un certain nombre de collègues pour leurs efforts productifs et analytiques considérables ainsi que leur contribution intellectuelle dans l'élaboration du texte et des tableaux, graphiques et cartes et dans la sélection des données présentées: Wendy Baldwin, John Bongaarts, Judith Bruce, Satvika Chalasani, Judy Diers, Gina Duclayan, Sarah Engebretsen, Rachel Goldberg, Nicole Haberland, Kelly Hallman, Robert Heidel, Paul Hewett, Laura Laski, Cynthia Lloyd, Ziad Mikati, Mark Montgomery, Jim Rosen, Christina Tse, Adam Weiner, et Sylvia Wong. Remerciements particuliers à Diana Graizbord et Marisela Morales pour leur dévouement exceptionnel et leur travail immense dans ce projet. On remercie aussi nos collègues d'Equilibres et Population pour la traduction en français.

Vos commentaires sont les bienvenus à l'adresse suivante : aweiner@popcouncil.org. 


\section{$\underline{\text { II - Notes techniques }}$}

1. Tous les tableaux de ce rapport ont été élaborés à partir de données collectées pour l'Enquête Démographique et de Santé du Niger 2006, menée par l'Institut National de la Statistique (INS) et le Ministère de la Santé Publique et de la Lutte contre les Endémies. L'EDS du Niger a sondé un échantillon représentatif de 7660 foyers. Les informations présentées dans les tableaux 1 à 8 et dans les graphiques et cartes correspondants proviennent de tous les résidents habituels au sein de la (ou des) tranche(s) d'âge des foyers sélectionnés. Les tableaux 9 à 26 et les graphiques et cartes correspondants sont issus d'un questionnaire individuel plus approfondi auquel ont répondu 9223 femmes âgées de 15 à 49 ans. Les données sont considérées comme représentatives au niveau national, urbain/rural et régional pour toutes les tranches d'âge.

Plus d'informations sur l'élaboration et les conclusions de l'EDS du Niger 2006 sont disponibles dans le rapport final (Institut National de la Statistique (INS) et Macro International Inc. 2007. Enquête Démographique et de Santé et à Indicateurs Multiples de Niger 2006. Calverton, Maryland, USA: INS et Macro International Inc.). Des informations sur les Enquêtes Démographiques et de Santé, y compris des rapports, d'autres publications et les données d'origine, sont disponibles sur le site web des EDS à http://www.measureedhs.com/.

2. Les tableaux indiquent les valeurs pour lesquelles des données sont disponibles dans l'EDS du Niger 2006. Les données sont segmentées en divers groupes pour autant qu'elles restent exactes. Des parenthèses autour d'un nombre signalent que la statistique se base sur 25 à 50 cas non pondérés et que la donnée devrait être considérée avec prudence. Un astérisque indique que la statistique se base sur moins de 25 individus et que la donnée a pour cette raison été omise.

3. Des cartes sont utilisées ponctuellement pour représenter les variations sousnationales ou régionales. Ces cartes répètent des informations présentées ailleurs sous forme de tableaux ou de graphiques car elles sont plus faciles à lire et sont particulièrement utiles pour les décideurs politiques représentant des circonscriptions sous-nationales. 


\section{III - Investir en faveur des adolescents : une stratégie pour la réalisation des Objectifs du Millénaire pour le développement}

Investir en faveur des adolescents, qui sont une composante économique et sociale vitale dans la plupart des sociétés, est fondamental dans le monde en développement, où une partie croissante de la population est âgée de moins de 24 ans. Par ailleurs, à mesure que le taux de fécondité diminue, beaucoup de pays se retrouve avec un "dividende démographique ", c'est-à-dire une hausse de la croissance économique qui survient quand une plus grande partie de la population est en âge de travailler. Lorsqu'un pays est capable d'employer ces travailleurs de manière productive, ces derniers peuvent fournir des ressources pour satisfaire les besoins des moins de 15 ans et des plus de 60 ans.

Les Objectifs du Millénaire pour le développement, définis récemment et largement réitérés (de même que la Convention sur l'élimination de toutes les formes de discrimination à l'égard des femmes et la Convention relative aux droits des enfants, adoptées en masse) fournissent un cadre de valeurs et d'actions souhaitables vis-à-vis des enfants et adolescents. Aucun des objectifs de ces accords ne peut être atteint sans un investissement considérable et de premier ordre dans les jeunes filles adolescentes et autres adolescents vulnérables :

- Une base économique forte ne peut pas être construite sans un renforcement des ressources socio-économiques des jeunes filles et des jeunes vulnérables et donc sans une rupture du cycle intergénérationnel de la pauvreté. Les femmes qui ont le contrôle de leurs ressources sont plus susceptibles que les hommes d'y faire appel pour améliorer la santé et l'éducation de leurs enfants et d'autres membres de leur famille. Investir en faveur des jeunes filles au moment le plus opportun n'est pas seulement une question de justice économique, mais bien d'efficacité économique.

- L'éducation primaire universelle est l'objectif minimum de la plupart des pays. La catégorie la plus démunie, dans presque tous les pays, est celle des jeunes filles rurales. Bien que les différences entre filles et garçons se réduisent, deux tiers des personnes qui n'ont jamais été scolarisées ou sont actuellement déscolarisées sont de sexe féminin. L'éducation des jeunes filles, en particulier la participation et la scolarisation au cours de l'adolescence, représente le meilleur investissement de « développement » en termes a) d'acquisition des compétences et des savoirs nécessaires à un travail productif, b) de retombées économiques, et c) de justice sociale. Les jeunes filles qui ont été scolarisées sont plus susceptibles d'éviter un mariage précoce et ont un meilleur avenir en matière de santé maternelle et infantile. Elles sont aussi davantage capables de - et enclines à investir dans la santé et l'éducation de leurs enfants, ce qui accroît particulièrement pour leurs filles les chances d'être scolarisées.

- Les efforts soutenus pour atteindre les jeunes filles avant la puberté sont des étapes cruciales dans la quête de l'égalité des sexes. Ils contribuent à prévenir les pires violations des droits de l'Homme (trafic, excision, travail domestique abusif, mariage précoce, relations sexuelles non souhaitées), des maladies ou affections souvent directement liées à une fécondité forte et non désirée, la mortalité maternelle, et l'infection à VIH. 
- Investir en faveur des jeunes filles les plus pauvres dans les pays les plus pauvres est susceptible de faire reculer les maladies et la mortalité maternelles. Les mêmes jeunes filles qui sont marginalisées du fait de leur exclusion scolaire et du fait qu'elles habitent dans des communautés pauvres, rurales et à minorité ethnique sont la cible de mariages précoces et de pratiques traditionnelles néfastes. Elles ont un accès limité aux services de santé et d'aide sociale et, en tant que mères d'un premier enfant, ont les plus forts risques de morbidité et mortalité maternelles.

- La population atteinte par l'épidémie du VIH est de plus en plus souvent jeune et féminine. Typiquement, les nouveaux rapports femmes/hommes d'incidence pour les 15-24 ans en Afrique subsaharienne sont de 3 pour 1, et parfois même jusqu'à 8 pour 1 dans certains pays. Sans des efforts soutenus, les jeunes filles marginalisées et désavantagées vont sans doute représenter une part de plus en plus grande et disproportionnée des cas d'infection à VIH.

- Dans les pays pauvres où les taux de fécondité sont élevés, l'âge au mariage et à la grossesse est un facteur déterminant pour la future croissance démographique. Les investissements en faveur des jeunes filles, pour promouvoir la scolarisation, protéger l'intégrité corporelle et encourager le mariage légal et consenti, pourraient améliorer de manière significative leur vie. De plus, cela pourrait réduire les fardeaux de leurs familles et de leurs communautés, et favoriser des diminutions dans le taux de croissance démographique. 


\section{IV - Résumé des données de l'EDS sur les adolescents}

\section{A - Cartographier la grande diversité des adolescents}

Les capacités et les perspectives des adolescents varient, souvent de manière considérable, selon l'âge, le sexe, le niveau de scolarité, la situation matrimoniale, la région et le milieu (urbain ou rural) de résidence et de naissance, et les appartenances culturelles. Comprendre cette diversité est essentiel pour élaborer des programmes et des politiques pour les adolescents pertinentes et ciblées. Beaucoup d'éléments sont en jeu si les programmes ne sont pas ciblés de façon adéquate. Les objectifs de ces programmes ne seront pas remplis si ces derniers ne touchent pas les populations les plus nécessiteuses. En fait, les programmes peuvent involontairement accroître les désavantages en fournissant des services à ceux qui en ont le moins besoin, tout en excluant ceux qui en ont le plus besoin. Les tableaux qui suivent mettent en relief les différences de base qu'il y a parmi les expériences et les perspectives des adolescents.

\section{Les jeunes urbains, ruraux et périurbains}

Les politiques de jeunesse (tant celles destinées à développer les compétences que celles censées réduire les effets de la pauvreté) doivent répondre aux environnements spécifiques dans lesquels les jeunes vivent. Une attention particulière doit être accordée aux différences de situations sociales et économiques entre les zones urbaines et rurales. Dans les villes et les grandes villes, les ressources pédagogiques et sanitaires clés sont plus facilement accessibles que dans les villages ruraux. Les grandes villes offrent en outre des perspectives d'activités génératrices de revenus plus diverses. Malgré cela, les jeunes (surtout ceux qui sont pauvres) sont loin d'être en mesure de bénéficier de ces ressources et perspectives urbaines. Les taux de scolarisation chez les populations urbaines pauvres sont beaucoup plus faibles que chez les résidents urbains plus riches. Dans de nombreux aspects de la santé, les populations urbaines pauvres s'en sortent à peine mieux que les habitants des villages ruraux. Pour certains, la diversité des niveaux de vie urbains a un aspect positif, car elle suggérerait qu'il est possible de s'élever dans l'échelle sociale. Mais pour beaucoup de jeunes filles et garçons pauvres, cette même diversité peut être interprétée très différemment, comme la preuve d'un fossé impossible à combler entre leurs situations et celles des élites urbaines. Les risques sociaux de la vie urbaine peuvent toucher aussi bien les jeunes pauvres que les mieux lotis, comme le montre clairement la hausse des taux urbains de VIH et de SIDA.

D'après les récentes estimations d'UN-Habitat ${ }^{1}$, le nombre de personnes habitant un bidonville atteint presque 1 milliard. Pourtant, les populations urbaines pauvres (surtout les adolescents pauvres) ont peu souvent été au centre des politiques de réduction de la pauvreté. Une attention particulière doit être accordée aux jeunes vivant en zones périurbaines, c'est-à-dire celles qui entourent les zones urbaines. Ces zones périurbaines se trouvent souvent en dehors de la sphère géographique des autorités locales et municipales, et peuvent donc être négligées en termes de prestations de services. On estime que ces zones sont parmi celles dont l'extension est la plus rapide dans le monde en développement.

\footnotetext{
${ }^{1}$ Programmes des Nations Unies pour les établissements humains (UN-Habitat). 2003. The challenge of slums: global report on human settlements. UN-Habitat : Nairobi.
} 
Il est possible dans environ la moitié des EDS récentes de localiser les quartiers où le sondage a eu lieu. En théorie, cette information pourrait être utilisée pour identifier les communautés périurbaines et les bidonvilles. Dans la plupart des cas cependant, il y a trop peu d'observations pour permettre aux chercheurs de caractériser de manière certaine les populations jeunes de ces communautés. Toutefois, on peut distinguer la jeunesse urbaine dans son ensemble de la jeunesse rurale, et accorder une attention particulière aux populations de foyers pauvres. Il est très possible que les migrants récents, surtout parmi les jeunes, vivent dans des situations instables sans services adéquats adaptés à la jeunesse. Pour comprendre les perspectives et les risques, on recommande de discerner les jeunes ruraux des jeunes urbains. Des distinctions supplémentaires sur la base des niveaux de vie est aussi souhaitable. Quand la taille des échantillons le permet, on peut analyser les EDS qui localisent leurs groupes d'échantillonnage, pour caractériser les environnements auxquels sont confrontés les adolescents des bidonvilles et les habitants des zones périurbaines. Il s'agit d'un domaine où plus de recherche est nécessaire et qui dépasse le cadre de cette publication.

\section{Tableau 1: Répartition de la population}

\begin{tabular}{|c|c|c|}
\hline \multicolumn{3}{|c|}{$\begin{array}{l}\text { Répartition des milieux de résidence } \\
\text { urbains ou ruraux et de la population } \\
\text { (en \%) }\end{array}$} \\
\hline & Urbain & Rural \\
\hline \multicolumn{3}{|l|}{ Filles } \\
\hline $10-14$ & 18.3 & 81.7 \\
\hline $15-19$ & 23.9 & 76.1 \\
\hline $20-24$ & 22.2 & 77.8 \\
\hline \multicolumn{3}{|l|}{ Garçons } \\
\hline $10-14$ & 18.0 & 82.0 \\
\hline $15-19$ & 28.5 & 71.5 \\
\hline $20-24$ & 32.0 & 68.0 \\
\hline
\end{tabular}

Lire la première colonne des données comme suit: « Le pourcentage des adolescentes/adolescents vivant en zones urbaines. » 


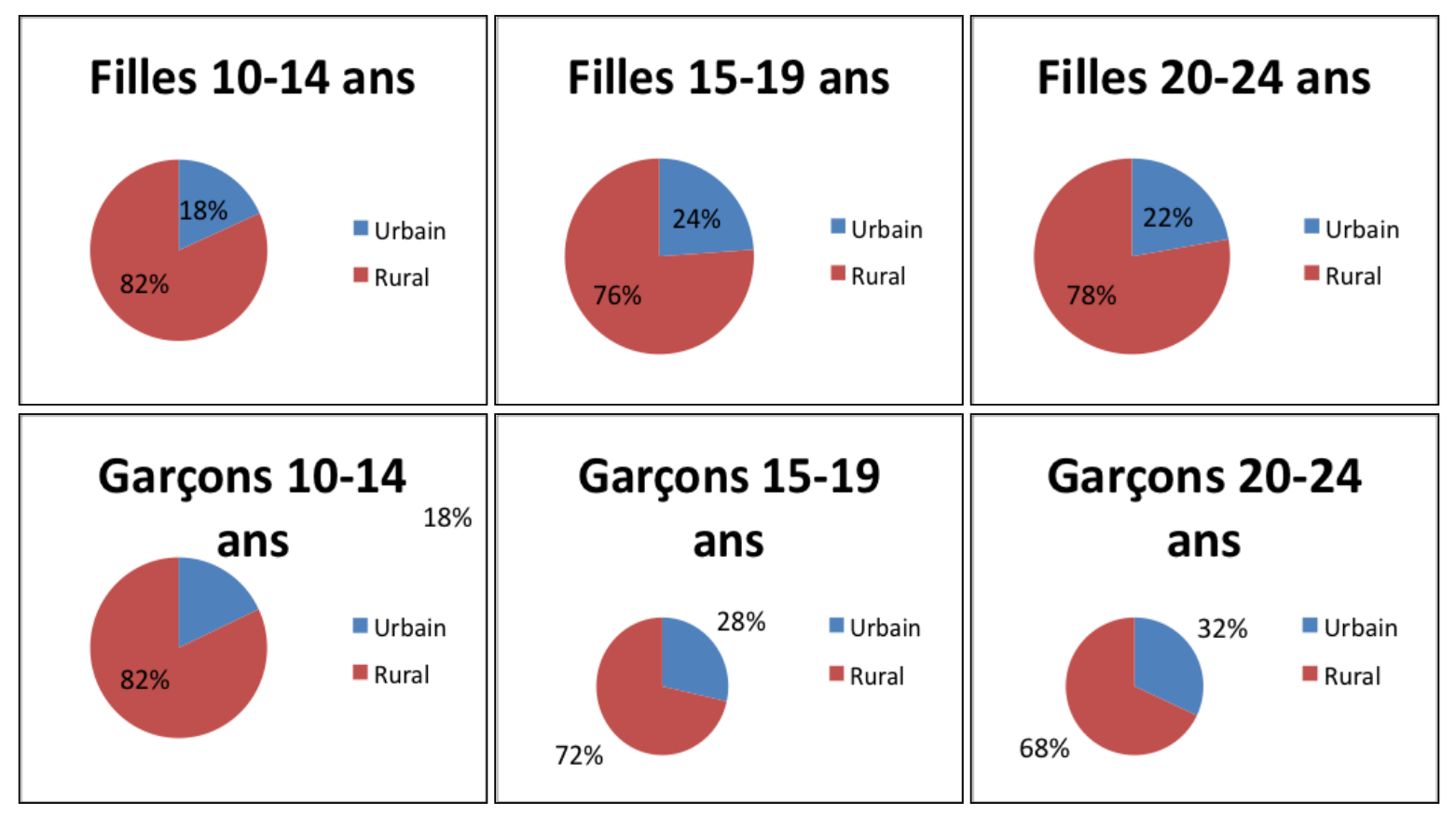

Présence des parents dans le foyer : vivre avec ses deux parents, un seul ou aucun

Beaucoup de politiques sociales, y compris celles de santé, supposent que les jeunes adolescents vivent avec au moins un de leurs parents, ou idéalement les deux. On présume qu'on peut compter sur les parents (ou même les grands-parents) pour apporter un revenu, un soutien moral ou logistique, un hébergement et une protection. Beaucoup de programmes destinés à cette tranche d'âge supposent un certain niveau d'engagement des parents et peuvent par conséquent chercher la contribution de ces derniers dans les politiques importantes.

Bien que la présence des deux parents n'assure pas qu'un jeune disposera de tous les atouts présumés, l'absence d'un des parents ou des deux peut, elle, être inquiétante. Les adolescents qui vivent sans l'un de leurs parents ou sans les deux peuvent être socialement isolés, ne pas posséder les ressources économiques nécessaires pour aller à l'école, et devoir supporter une activité génératrice de revenus tant pour eux-mêmes que pour les autres membres de la famille. L'absence partielle ou totale des parents peut réduire l'accès des adolescents aux soins et même la garantie de leur propre sécurité, des études ayant récemment confirmé que les adolescents orphelins ou doublement orphelins possèdent des taux de VIH plus élevés. Aux vues de ces désavantages, certains pays ont commencé à se pencher sur les droits des sous-catégories de population désavantagées et à envisager des politiques allant dans ce sens, dont des programmes de transfert d'espèces aux enfants désavantagés, la priorité donnée aux personnes du quintile des faibles revenus vivant avec un seul (ou aucun) parent, et les Individual Development Accounts (comptes d'épargne) pour enfants orphelins. Ainsi, les informations sur la présence des parents dans le foyer sont un facteur important pour réfléchir sur le ciblage des ressources. Il est aussi utile de noter les variations significatives entre régions sous-nationales et entre les jeunes garçons et filles. Quand 
les jeunes adolescents de 10-14 ans vivent avec un seul parent, c'est presque toujours avec leur mère et non avec leur père. Ainsi, les désavantages de la mère (en revenu, protection et pouvoir social, entre autres) peuvent se traduire par des fardeaux et des risques particuliers pour les enfants, et surtout les jeunes filles.

Tableau 2 : Présence des parents dans le foyer

\begin{tabular}{|c|c|c|c|c|c|c|c|c|}
\hline \multicolumn{9}{|c|}{ Présence des parents dans le foyer chez les jeunes de 10-14 ans (en \%) } \\
\hline \multirow[t]{2}{*}{ Région } & \multicolumn{2}{|c|}{$\begin{array}{l}\text { Présence des } \\
\text { deux parents }\end{array}$} & \multicolumn{2}{|c|}{$\begin{array}{l}\text { Présence de la } \\
\text { mère seulement }\end{array}$} & \multicolumn{2}{|c|}{$\begin{array}{c}\text { Présence du } \\
\text { père seulement }\end{array}$} & \multicolumn{2}{|c|}{$\begin{array}{l}\text { Absence des } \\
\text { deux parents }\end{array}$} \\
\hline & Filles & Garçons & Filles & Garçons & Filles & Garçons & Filles & Garçons \\
\hline Agadez & 65.4 & 63.4 & 12.1 & 11.6 & 8.8 & 8.8 & 13.7 & 16.2 \\
\hline Diffa & 59.5 & 68.1 & 13.1 & 10.4 & 7.8 & 9.4 & 19.6 & 12.1 \\
\hline Dosso & 56.0 & 60.6 & 23.6 & 23.1 & 4.5 & 5.3 & 16.0 & 11.0 \\
\hline Maradi & 77.5 & 80.4 & 10.0 & 6.7 & 3.9 & 6.9 & 8.6 & 6.0 \\
\hline Tahoua & 50.4 & 59.4 & 26.2 & 21.6 & 8.2 & 7.4 & 15.3 & 11.6 \\
\hline Tillabéri & 55.9 & 69.3 & 19.4 & 15.4 & 6.4 & 6.1 & 18.3 & 9.0 \\
\hline Zinder & 59.1 & 63.5 & 10.0 & 3.2 & 8.8 & 13.9 & 22.0 & 19.5 \\
\hline Niamey & 55.7 & 66.1 & 13.5 & 14.2 & 6.0 & 5.7 & 24.6 & 14.1 \\
\hline Urbaine & 56.0 & 61.6 & 13.5 & 12.9 & 8.7 & 9.0 & 21.7 & 16.5 \\
\hline Rurale & 60.9 & 68.1 & 17.5 & 13.2 & 6.1 & 7.9 & 15.6 & 10.8 \\
\hline $\begin{array}{l}\text { Niveau } \\
\text { national }\end{array}$ & 60.0 & 66.9 & 16.8 & 13.2 & 6.5 & 8.1 & 16.7 & 11.8 \\
\hline
\end{tabular}

Lire la première colonne des données comme suit : « Le pourcentage des filles de 10-14 ans qui habitent avec leurs deux parents. » 


\section{Graphique 2 : Présence des parents dans le foyer chez les 10-14 ans (en \%)}

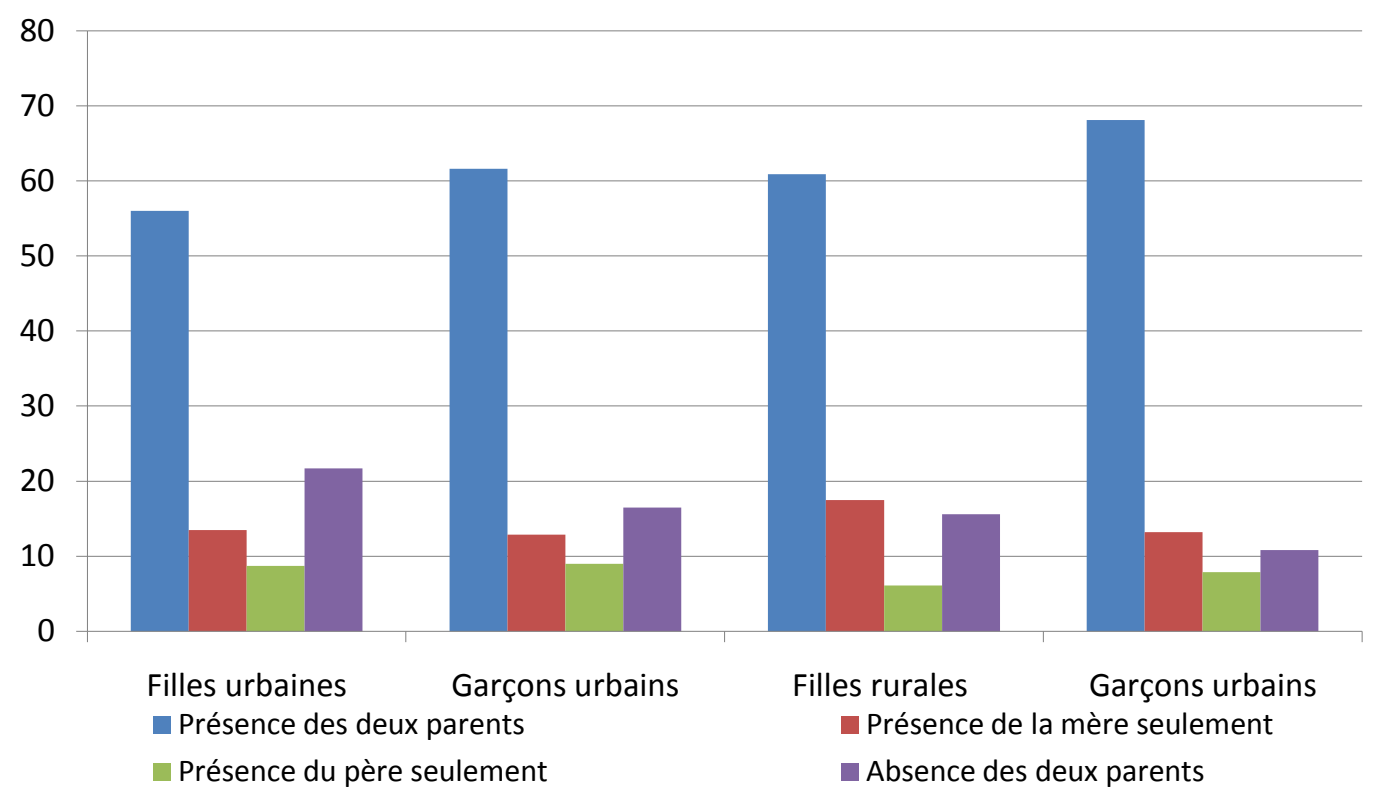

Tableau 3 : Survie des parents

\begin{tabular}{|c|c|c|c|c|c|c|c|c|}
\hline \multicolumn{9}{|c|}{ Survie des parents parmi les $10-14$ ans (en \%) } \\
\hline \multirow[t]{2}{*}{ Région } & \multicolumn{2}{|c|}{$\begin{array}{l}\text { Deux parents en } \\
\text { vie }\end{array}$} & \multicolumn{2}{|c|}{$\begin{array}{l}\text { Mère en vie } \\
\text { uniquement }\end{array}$} & \multicolumn{2}{|c|}{$\begin{array}{c}\text { Père en vie } \\
\text { uniquement }\end{array}$} & \multicolumn{2}{|c|}{$\begin{array}{l}\text { Aucun des deux } \\
\text { parents en vie }\end{array}$} \\
\hline & Filles & Garçons & Filles & Garçons & Filles & Garçons & Filles & Garçons \\
\hline Agadez & 91.9 & 89.5 & 5.0 & 6.2 & 2.6 & 4.4 & 0.5 & 0.0 \\
\hline Diffa & 89.7 & 86.0 & 5.5 & 7.9 & 4.5 & 5.8 & 0.4 & 0.2 \\
\hline Dosso & 87.1 & 88.6 & 9.1 & 7.4 & 3.2 & 3.2 & 0.7 & 0.8 \\
\hline Maradi & 95.6 & 92.1 & 3.6 & 2.6 & 0.5 & 4.8 & 0.3 & 0.5 \\
\hline Tahoua & 86.1 & 88.8 & 6.6 & 6.4 & 5.3 & 3.5 & 2.0 & 1.3 \\
\hline Tillabéri & 88.8 & 91.9 & 6.2 & 4.6 & 4.4 & 3.1 & 0.5 & 0.4 \\
\hline Zinder & 89.1 & 89.2 & 5.8 & 3.1 & 4.6 & 6.5 & 0.6 & 1.3 \\
\hline Niamey & 85.9 & 87.8 & 7.5 & 8.3 & 2.0 & 3.0 & 4.5 & 0.9 \\
\hline Urbaine & 88.6 & 89.6 & 6.4 & 6.4 & 2.4 & 2.8 & 2.5 & 1.2 \\
\hline Rurale & 89.5 & 90.0 & 6.1 & 4.6 & 3.7 & 4.6 & 0.7 & 0.8 \\
\hline $\begin{array}{c}\text { Niveau } \\
\text { national }\end{array}$ & 89.3 & 89.9 & 6.2 & 5.0 & 3.5 & 4.3 & 1.0 & 0.9 \\
\hline
\end{tabular}

Lire la première colonne des données comme suit: « Le pourcentage des filles de 10-14 ans dont les deux parents sont en vie. » 


\section{Graphique 3 : Survie des parents parmi les 10-14 ans (en \%)}

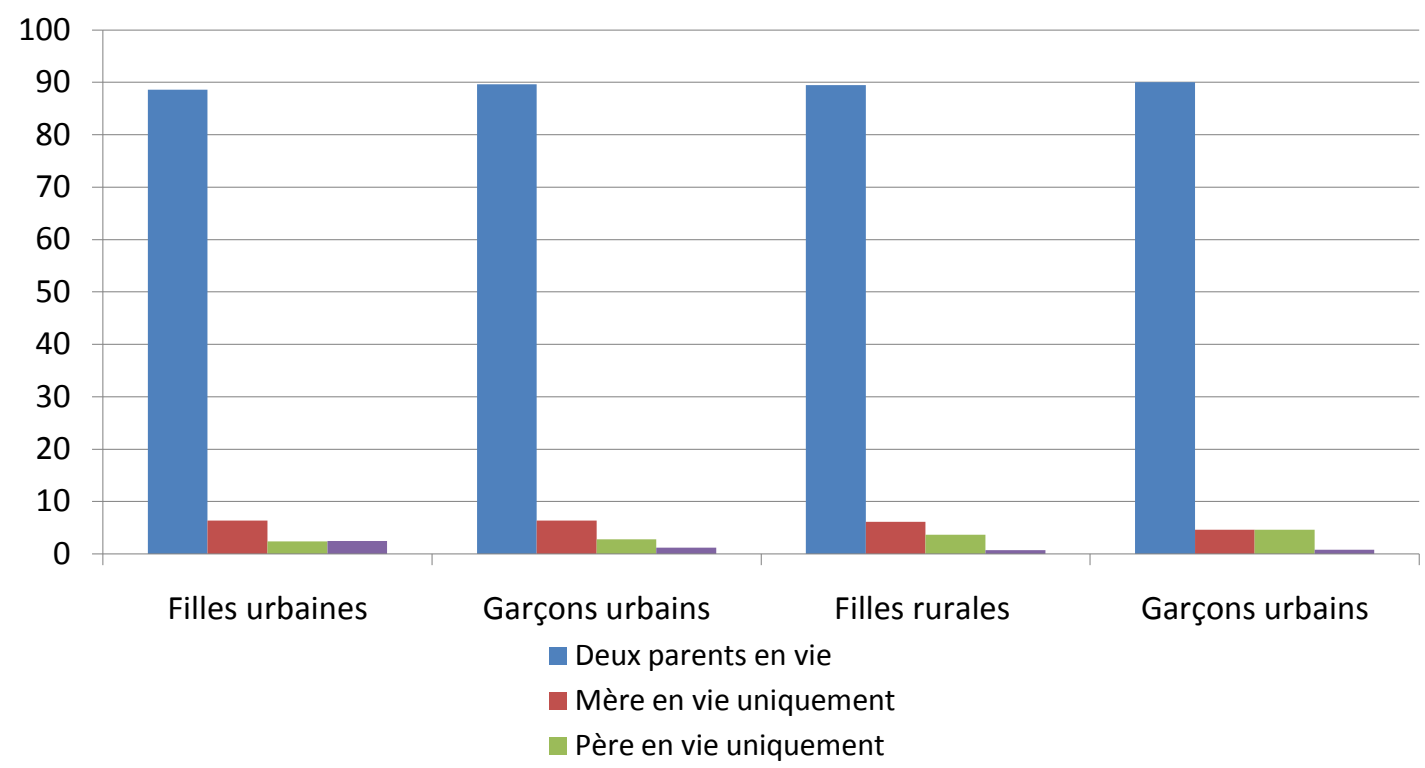

\section{La scolarisation pendant l'adolescence: une stratégie de santé et de développement clé}

L'éducation est un facteur essentiel pour une transition saine vers l'âge adulte. Pendant l'enfance et l'adolescence, le processus d'apprentissage a un rôle plus intense que pendant les autres phases de la vie. Au cours de l'adolescence, les jeunes développent des capacités physiques et cognitives et acquièrent le savoir et les connaissances nécessaires pour devenir des adultes en bonne santé et productifs. Apporter une éducation de qualité dans un environnement sûr et maintenir les enfants à l'école est une stratégie transversale, qui relie de multiples priorités de développement. On a par exemple établi un lien entre le fait d'être scolarisé et des reculs de l'âge du premier rapport sexuel, du mariage et de la grossesse. Des politiques et programmes adéquats et ciblés, qui contribuent à maintenir les jeunes scolarisés tout au long de l'adolescence et à les rattacher au réseau social représenté par les écoles, peuvent avoir des effets considérables sur leur développement personnel et minimiser leurs vulnérabilités face aux défis de l'environnement extrascolaire.

La scolarisation est le droit des adolescents le plus largement admis et défini, et cela se reflète dans les décisions politiques que les gouvernements prennent en faveur des adolescents. Naturellement, les décideurs politiques soucieux des questions de santé de la procréation ainsi que de la prévention et de la gestion du VIH ont consacré beaucoup d'attention aux programmes scolaires de prévention du VIH. Néanmoins, bien qu'il soit évidemment important de cibler les jeunes dans les écoles, peu d'attention a jusqu'ici été accordée aux risques de santé de la procréation (souvent élevés) des adolescents non scolarisés, et en particulier les jeunes filles. Cela a conduit à un manque de programmes actifs ciblant ces derniers.

Les études qui portent sur la scolarisation et son rapport avec les comportements de santé de la procréation se sont souvent focalisés sur les liens entre le nombre d'année de scolarité d'une part, et le recul dans le temps du mariage et du premier enfant, une 
fécondité plus basse et des revenus plus élevés dans le marché du travail d'autre part². Une récente étude portant sur 48 pays (dont une majorité à faible revenu), conduite par Cynthia Lloyd, s'est penchée sur les bienfaits de la scolarisation pendant l'adolescence sur la santé de la procréation des jeunes filles et garçons, quelle que soit la réussite scolaire, la qualité de l'école (mesurée sur la compétence des élèves) ou le rapport entre âge et niveau ${ }^{3}$. Cette étude de Lloyd, qui fait appel aux Enquêtes Démographiques et de Santé récemment publiées, s'est focalisée sur le comportement procréatif d'adolescents de 15-17 ans scolarisés. Sa découverte la plus importante est sans doute celle qui concerne la proportion de jeunes filles de cette tranche d'âge qui indiquent avoir déjà eu un rapport sexuel. Les jeunes filles scolarisées (presque toutes non mariées) sont beaucoup moins susceptibles d'indiquer avoir eu des rapports sexuels que les jeunes filles non scolarisées (aussi bien mariées que non mariées). Cela vaut même pour l'Afrique subsaharienne, où une large proportion des 15-17 ans qui sont scolarisés étudie au niveau primaire. L'augmentation du taux d'adolescents scolarisés ne semble pas réduire la force du constat d'un lien entre la scolarisation et une meilleure santé de la procréation. Il s'avère que les adolescents scolarisés, et en particulier les jeunes filles, sont mieux informés et motivés pour éviter les infections sexuellement transmissibles et les grossesses ${ }^{4}$. Les jeunes filles scolarisées sont moins susceptibles que les jeunes filles non scolarisées de leur âge d'avoir déjà eu un rapport sexuel; de plus, si elles ont une activité sexuelle, elles sont plus susceptibles de faire appel à une méthode de contraception. Le risque d'abandonner l'école s'accroît considérablement pour les jeunes filles qui ont déjà eu un rapport sexuel avant le mariage ${ }^{5}$. Par ailleurs, les jeunes filles qui sont actuellement scolarisées, et qui ont eu peu ou pas d'interruptions dans leur parcours scolaire, sont moins susceptibles de tomber enceintes que les jeunes filles qui ont eu des interruptions de scolarité. Elles sont aussi moins susceptibles d'abandonner l'école si elles tombent effectivement enceintes ${ }^{6}$.

\footnotetext{
${ }^{2}$ Summers, Lawrence. (1994) Investing in All the People, Document de séminaire de EDI n 45, Institut de développement économique (EDI) de la Banque mondiale ; Odaga, Adhiambo et Ward Heneveld (1995). "Girls and Schools in Sub-Saharan Africa," Document technique de la Banque mondiale 298.

3 Lloyd, Cynthia B. 2006. "Schooling and adolescent reproductive behavior in developing countries." Document commandé par le United Nations Millennium Project pour le rapport Public Choices, Private Decisions: Sexual and Reproductive Health and the Millennium Development Goals. New York: UN Millennium Project.

${ }^{4}$ Lloyd, Cynthia B. 2008. "The role of schools in promoting sexual and reproductive health among adolescents in developing countries," in S. Malarcher (ed.), Social Determinants of Sexual and Reproductive Health: Informing Future Research and Programme Needs. Genève : Organisation mondiale de la Santé.

${ }^{5}$ Ibid.

${ }^{6}$ Grant, Monica et Kelly Hallman. 2006. "Pregnancy-related school dropout and prior school performance in South Africa," Policy Research Division Working Paper ${ }^{\circ}{ }^{212}$. New York : Population Council.
} 
Tableau 4A : Scolarisation des 10-14 ans

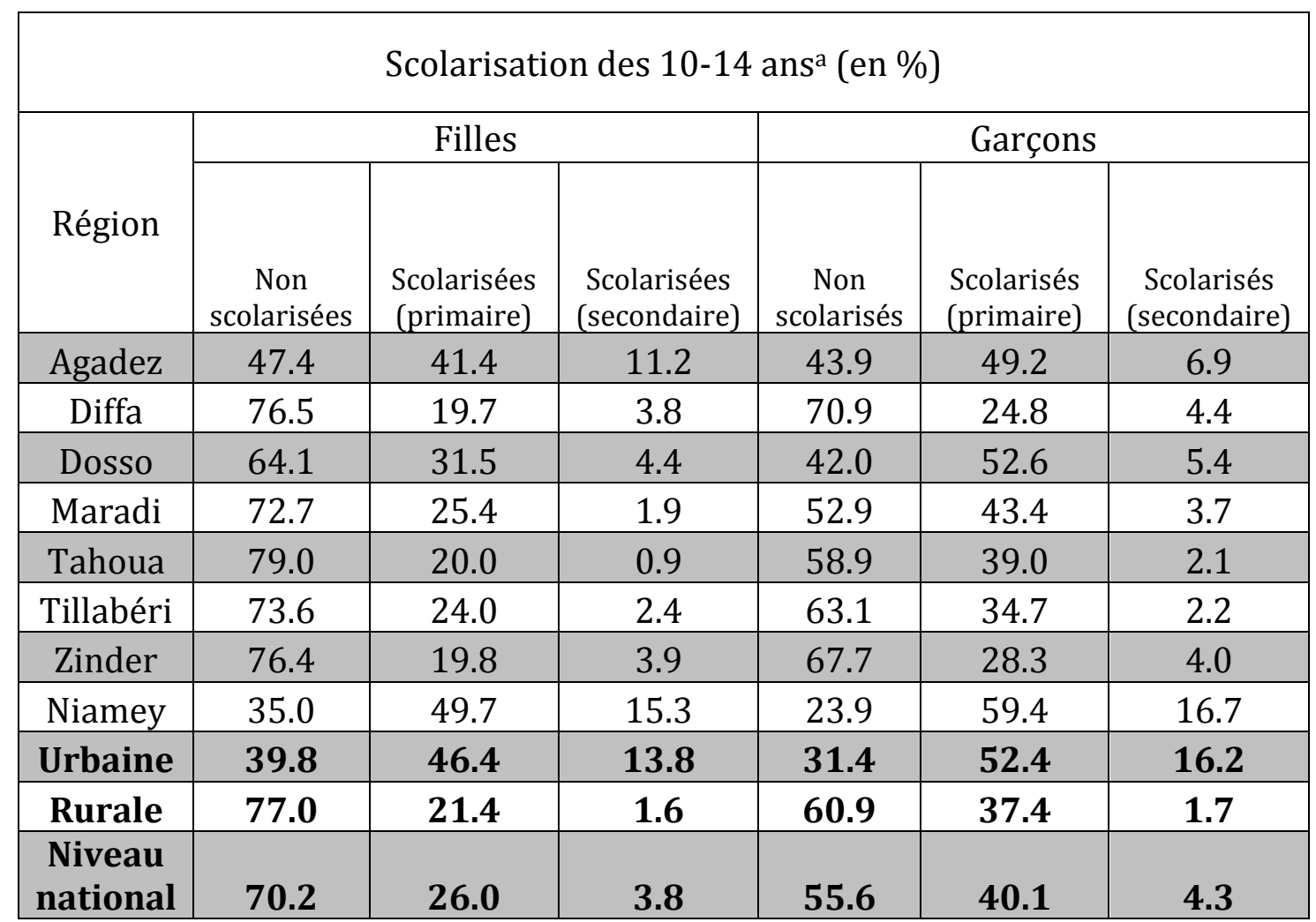

Lire la première colonne des données comme suit: « Le pourcentage des filles de 10-14 ans non-scolarisées. »

a Scolarisation à n'importe quel moment pendant l'année scolaire actuelle. 
Tableau 4B : Scolarisation des 15-19 ans

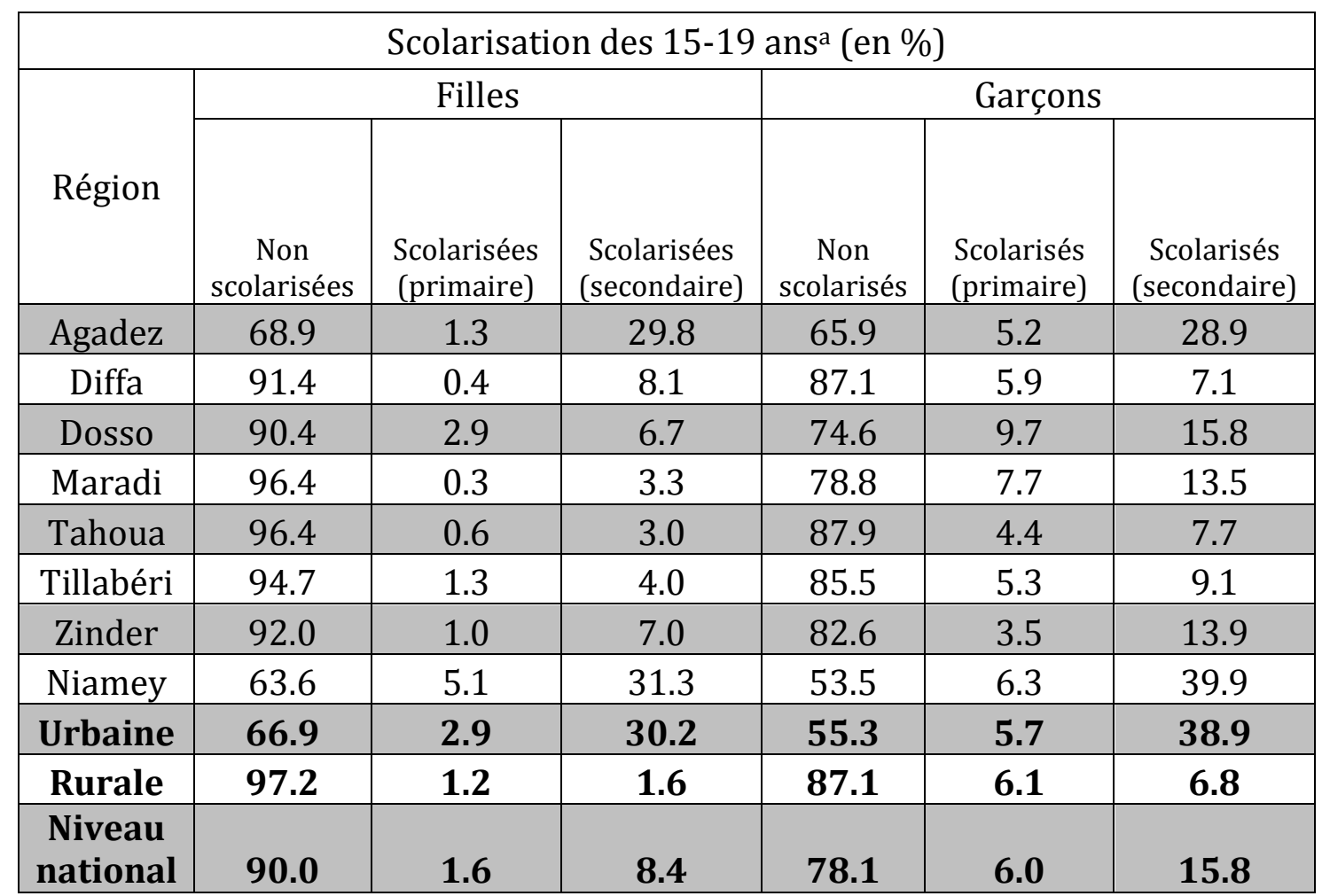

Lire la première colonne des données comme suit: «Le pourcentage des filles de 15-19 ans non-scolarisées. »

${ }^{a}$ Scolarisation à n'importe quel moment pendant l'année scolaire actuelle. 

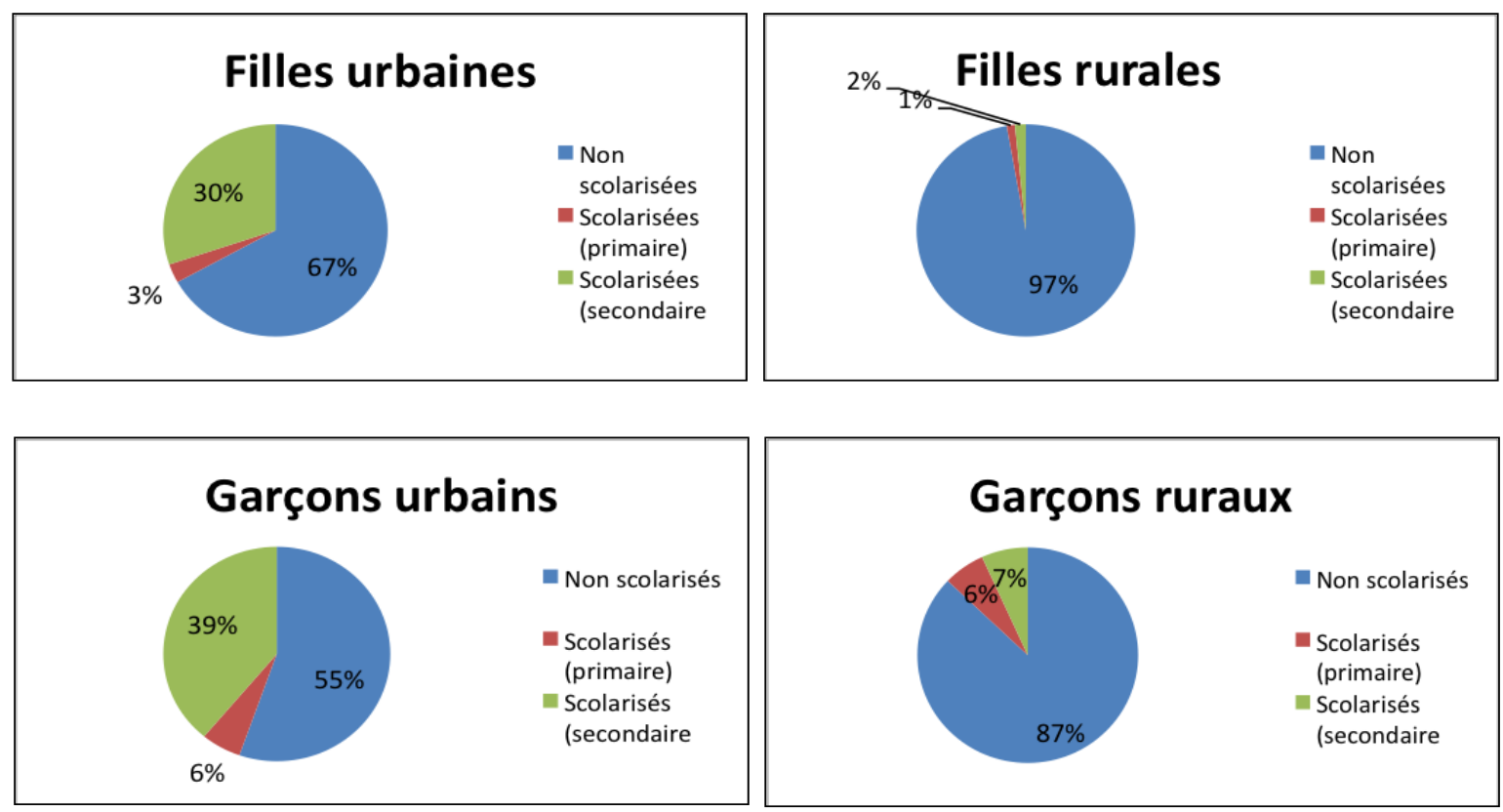

\section{Les filles mariées : une catégorie négligée}

Dans les pays en développement, la majorité des jeunes filles de 15-19 ans qui ont une activité sexuelle sont mariées. Le mariage précoce reste une pratique courante dans les régions en grande partie rurales d'Asie du Sud et d'Amérique latine, et, de manière frappante, dans beaucoup de pays d'Afrique subsaharienne où le taux de prévalence du VIH dépasse les $5 \%$ en ce qui concerne les femmes qui fréquentent les cliniques prénatales (l'épidémie étant définie comme généralisée et ayant atteint sa phase d'état). Si les tendances actuelles continuent, 100 millions de jeunes filles seront mariées avant l'âge de 18 ans au cours des dix prochaines années, et environ 1 sur 7 parmi elles le seront avant l'âge de 15 ans (analyse du Population Council à partir des données de pays des EDS et de l'ONU, en 2006).

Les jeunes filles mariées, en tant que catégorie distincte, ont peu suscité l'attention des politiques sociales et sanitaires, qui regroupent généralement toutes les femmes mariées ensemble quelque soit leur âge, leur âge au mariage et les caractéristiques de la relation maritale. De même, les initiatives destinées à servir les jeunes ont négligé aussi bien les jeunes filles risquant un mariage précoce que celles mariées, en se focalisant principalement sur les populations non mariées, souvent scolarisées.

Autre point très préoccupant: les risques encourus par les jeunes filles veuves, divorcées ou abandonnées du fait de la nature de leur mariage (son inégalité, ou une mort du mari par le $\mathrm{VIH}$ ), ainsi que l'exclusion sociale et les risques économiques immenses qu'elles subissent après le mariage.

Les jeunes filles mariées font face à une multitude d'autres défis qui limitent leur capacité à protéger leur santé et leur bien-être. À leur mariage, elles subissent souvent 
une réduction soudaine de la taille de leurs réseaux sociaux, ce qui les laisse avec peu d'amis, voire aucun. Cette isolation sociale peut les couper de certains droits essentiels (et garantis constitutionnellement dans beaucoup de pays). En outre, elles ont généralement un niveau d'études bas et aucune opportunité de scolarisation, un contrôle limité des ressources, un pouvoir de mobilité très restreint et peu ou pas de pouvoir dans leur nouveau foyer ${ }^{7}$. Ainsi, elles peuvent être confrontées à des défis considérables vis-à-vis de la négociation de relations sexuelles protégées. Une des composantes clés d'une politique efficace est de se pencher sur le mariage précoce là où il existe, de créer des programmes qui améliorent la sécurité du mariage dans tous les cas, et d'apporter des soutiens appropriés aux jeunes mariées et aux mères d'un premier enfant les plus jeunes. Voir le tableau 12 pour des données sur le mariage précoce.

Tableau 5 : Situation matrimoniale chez les filles

\begin{tabular}{|c|c|c|c|c|c|c|}
\hline \multirow{2}{*}{ Situation matrimoniale chez les filles de 15-24 ans (en \%) } \\
\hline \multirow{3}{*}{ Région } & \multicolumn{2}{|c|}{$\begin{array}{c}\text { Actuellement } \\
\text { mariées ou en } \\
\text { union }\end{array}$} & \multicolumn{2}{c|}{$\begin{array}{c}\text { Séparées, } \\
\text { divorcées ou } \\
\text { veuves }\end{array}$} & \multicolumn{2}{c|}{ Jamais mariées } \\
\cline { 2 - 8 } & $\begin{array}{c}15-19 \\
\text { ans }\end{array}$ & $\begin{array}{c}20-24 \\
\text { ans }\end{array}$ & $\begin{array}{c}15-19 \\
\text { ans }\end{array}$ & $\begin{array}{c}20-24 \\
\text { ans }\end{array}$ & $\begin{array}{c}15-19 \\
\text { ans }\end{array}$ & $\begin{array}{c}20-24 \\
\text { ans }\end{array}$ \\
\hline Agadez & 20.9 & 61.2 & 3.6 & 11.6 & 75.5 & 27.2 \\
\hline Diffa & 67.3 & 88.5 & 2.4 & 8.0 & 30.3 & 3.5 \\
\hline Dosso & 55.4 & 89.1 & 0.6 & 3.5 & 44.0 & 7.4 \\
\hline Maradi & 84.6 & 97.7 & 0.6 & 0.9 & 14.8 & 1.4 \\
\hline Tahoua & 64.5 & 91.7 & 0.9 & 4.5 & 34.6 & 3.8 \\
\hline Tillabéri & 53.6 & 88.4 & 1.6 & 1.9 & 44.8 & 9.7 \\
\hline Zinder & 69.5 & 93.0 & 4.5 & 3.4 & 26.0 & 3.6 \\
\hline Niamey & 16.4 & 47.4 & 1.6 & 3.6 & 82.1 & 49.1 \\
\hline Urbaine & $\mathbf{2 3 . 1}$ & $\mathbf{5 9 . 7}$ & $\mathbf{2 . 2}$ & $\mathbf{5 . 0}$ & $\mathbf{7 4 . 7}$ & $\mathbf{3 5 . 4}$ \\
\hline Rurale & $\mathbf{7 0 . 4}$ & $\mathbf{9 3 . 9}$ & $\mathbf{1 . 5}$ & $\mathbf{2 . 9}$ & $\mathbf{2 8 . 1}$ & $\mathbf{3 . 2}$ \\
\hline Niveau national & $\mathbf{5 9 . 0}$ & $\mathbf{8 6 . 4}$ & $\mathbf{1 . 7}$ & $\mathbf{3 . 3}$ & $\mathbf{3 9 . 3}$ & $\mathbf{1 0 . 3}$ \\
\hline
\end{tabular}

Lire la première colonne des données comme suit: « Le pourcentage des filles de 15-19 ans mariées ou vivant en union. »

\footnotetext{
7 Haberland, Nicole, Erica Chong, et Hillary J. Bracken. 2003. "Married Adolescents: An Overview," document préparé pour la Consultation technique sur les adolescentes mariées de l'OMS, I'UNFPA et le Population Council. OMS : Genève.
} 
Graphique 5 : Situation matrimoniale chez les filles, par région

Filles de 15-19 ans

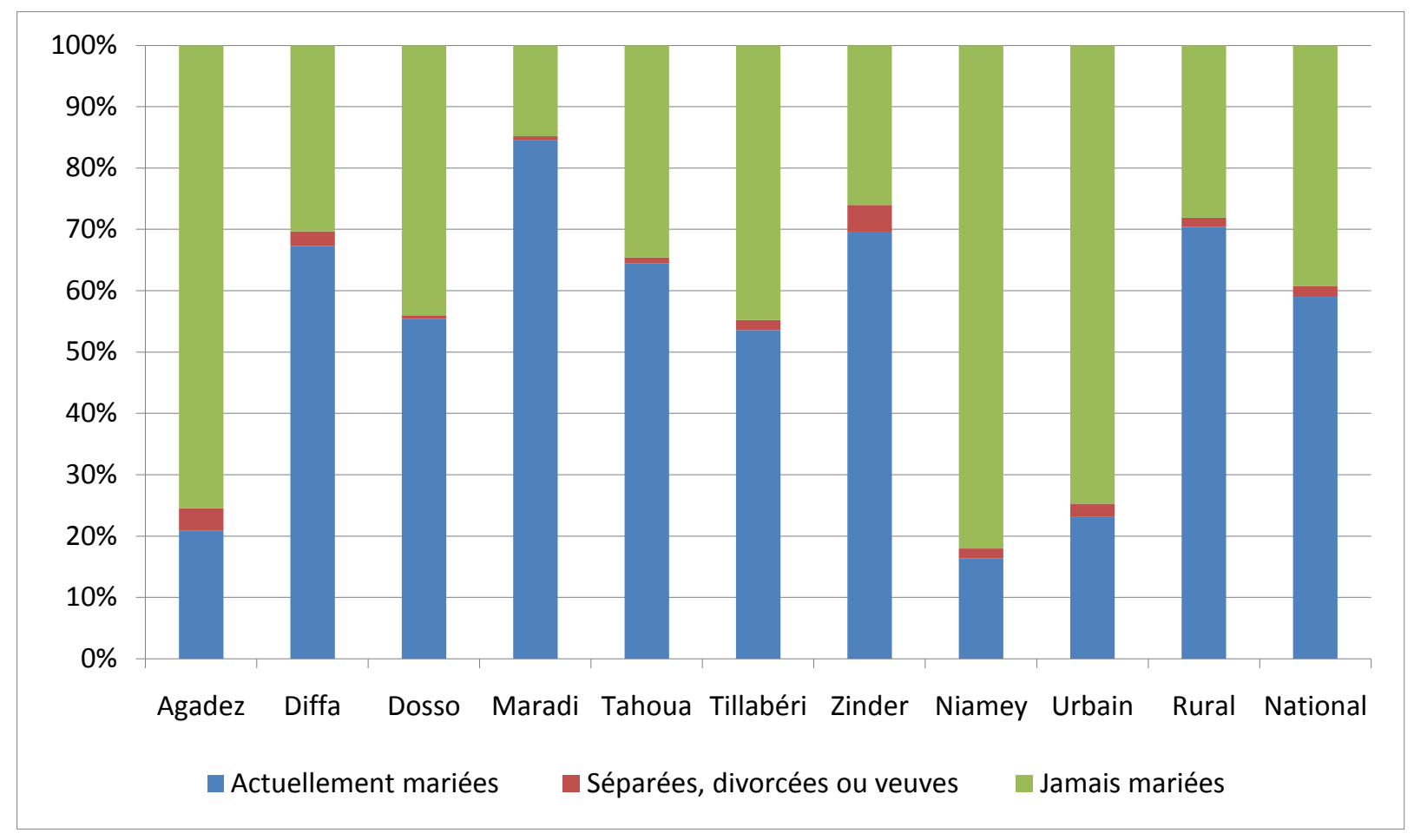

Filles de 20-24 ans

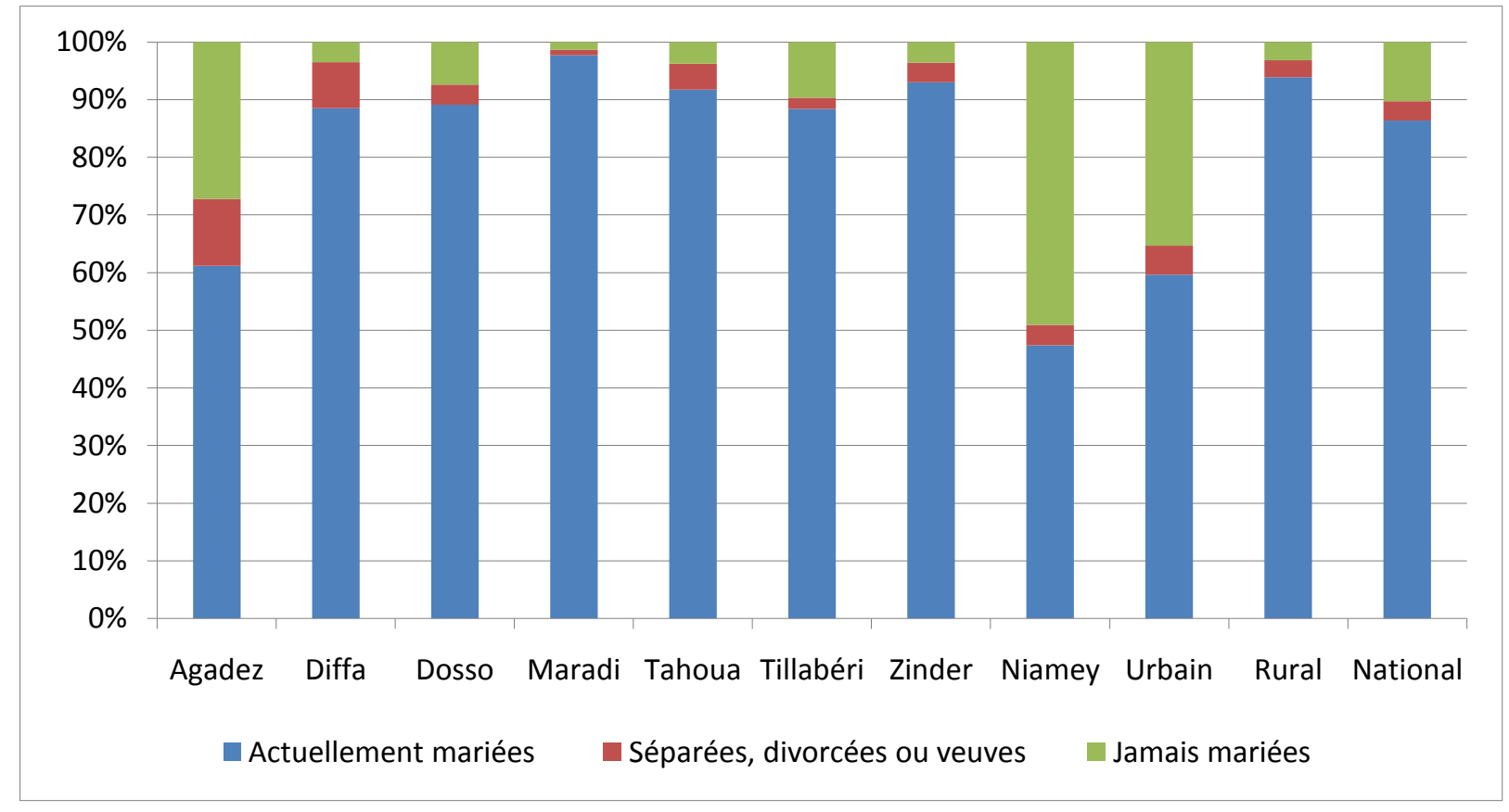




\section{B - Les transitions importantes qui désavantagent les très jeunes adolescents}

Les très jeunes adolescents (les enfants âgés de 10 à 14 ans) subissent des changements physiques, émotionnels, sociaux et intellectuels extrêmement importants. Durant cette période, de nombreux très jeunes adolescents arrivent à l'âge de la puberté, ont leurs premières expériences sexuelles, et, dans le cas des jeunes filles, peuvent même être mariés prématurément. Dans la majorité des cas, le début de l'adolescence se caractérise par une bonne santé et un environnement familial stable, mais il peut également être un moment où l'enfant est vulnérable car il doit faire face à des modifications radicales et très rapides de sa situation, et doit assumer un rôle nouveau, endosser des responsabilités, travailler, devenir chef de famille, et s'occuper des siens. Dans de nombreux pays, l'impact du VIH, de la pauvreté, des conflits politiques et sociaux sur les familles et les communautés a affaibli les systèmes de protection sociale traditionnels et accru la vulnérabilité des jeunes adolescents.

Il est donc essentiel que les décideurs et les personnes chargées de mettre en place des programmes tiennent compte tant des perspectives particulières qui s'ouvrent aux très jeunes adolescents que de leur vulnérabilité. Dans la plupart des pays, des politiques existent pour que les enfants de cet âge soient scolarisés, et leur taux de scolarisation est effectivement plus élevé que celui des adolescents plus âgés. Il est essentiel de tirer parti de cette situation. Cependant, il est également important de noter que de nombreux adolescents ne sont pas scolarisés et courent donc un risque accru de rencontrer des difficultés. Outre le fait qu'ils sont négligés d'un point de vue éducatif, les très jeunes adolescents sont particulièrement négligés par les décideurs et ne sont pas touchés par les programmes de santé infantile et maternelle et par les programmes destinés à donner plus d'autonomie aux femmes.

Il est important d'en savoir davantage sur le moment où s'effectuent ces transitions importantes, sur leur nature exacte, et sur les conséquences qu'elles ont pour les jeunes adolescents, et en particulier pour les groupes les plus vulnérables. Mettre en place des programmes pour les jeunes de cet âge, c'est reconnaître la capacité des jeunes à évoluer. Si l'on veut créer des conditions favorables aux jeunes adolescents, il convient que les interventions aient lieu à des moments clés de leur existence, avant que leur mode de vie ne soit fixé.

\section{Soutenir les jeunes les plus vulnérables}

Comme nous l'avons indiqué précédemment, les jeunes gens entre 10 et 14 ans sont à une période de transition : ils quittent l'enfance et entrent à l'âge de la puberté. Cette période s'accompagne en général de responsabilités accrues au sein de la famille, et les jeunes filles subissent en particulier des pressions sociales pour se marier, avoir des relations sexuelles et des enfants. Le jeune de cet âge qui ne vit pas avec ses parents et n'est pas scolarisé court un risque important. Il est très probable qu'il ne reçoive aucun soutien de la part de sa famille ou de ses pairs pour faire face aux difficultés qui se présentent à lui, et qu'on ne lui offre pas les moyens de devenir un membre productif de la société. Dans certains cas de figure, il s'agit de très jeunes adolescentes qui sont des domestiques, ont quitté les zones rurales pour trouver du travail et avoir accès à une formation, ou ont fui un mariage forcé. Dans d'autres cas, elles sont été mariées très jeunes et vivent désormais avec leur conjoint et peut-être même avec leur belle-famille. Ces catégories de jeunes filles sont parmi les moins susceptibles de faire appel aux 
services sociaux ou d'être sollicitées par eux, et il est donc très important de mettre en place en amont des règles pour diminuer le risque qu'elles soient exploitées.

Tableau 6 : Adolescents non-scolarisés et ne vivant avec aucun des parents

\begin{tabular}{|c|c|c|}
\hline \multicolumn{2}{|c|}{$\begin{array}{c}\text { Pourcentage des adolescents de } 10-14 \text { ans qui ne } \\
\text { sont pas scolarisés et ne vivent pas avec l'un ou } \\
\text { l'autre parent }\end{array}$} \\
\hline Région & Filles & Garçons \\
\hline Agadez & 6.7 & 7.4 \\
\hline Diffa & 14.4 & 9.6 \\
\hline Dosso & 12.5 & 4.7 \\
\hline Maradi & 7.3 & 3.7 \\
\hline Tahoua & 12.0 & 4.8 \\
\hline Tillabéri & 14.9 & 4.9 \\
\hline Zinder & 16.5 & 14.0 \\
\hline Niamey & 14.9 & 5.3 \\
\hline Urbain & $\mathbf{1 1 . 2}$ & $\mathbf{6 . 0}$ \\
\hline Rurale & $\mathbf{1 3 . 0}$ & $\mathbf{6 . 8}$ \\
\hline Niveau national & $\mathbf{1 2 . 7}$ & $\mathbf{6 . 6}$ \\
\hline
\end{tabular}

Lire la première colonne des données comme suit : « Le pourcentage des filles de 10-14 ans non-scolarisées et ne vivant avec aucun des parents. » 
Graphique 6 : Adolescents de 10-14 ans non scolarisés et ne vivant avec aucun des parents

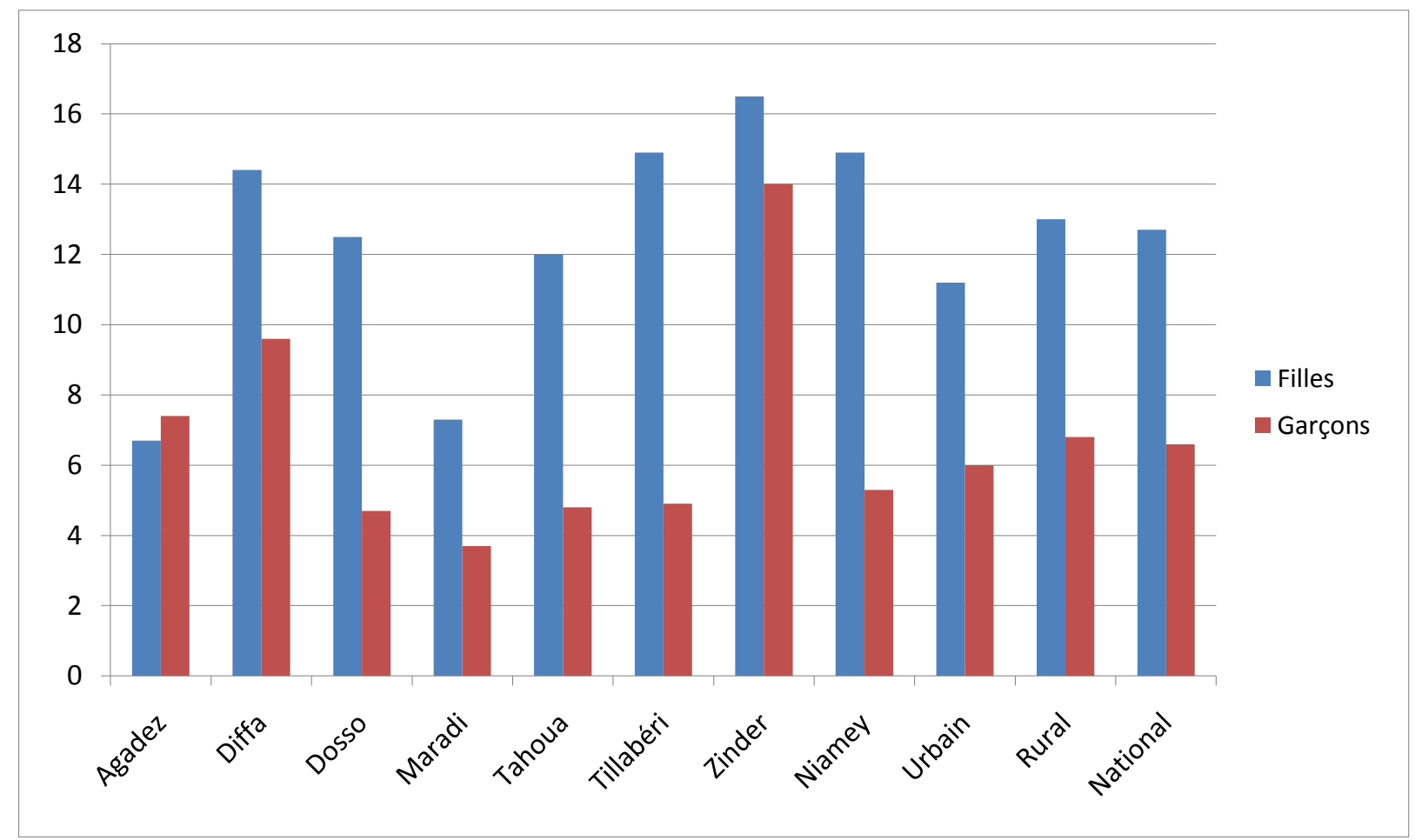




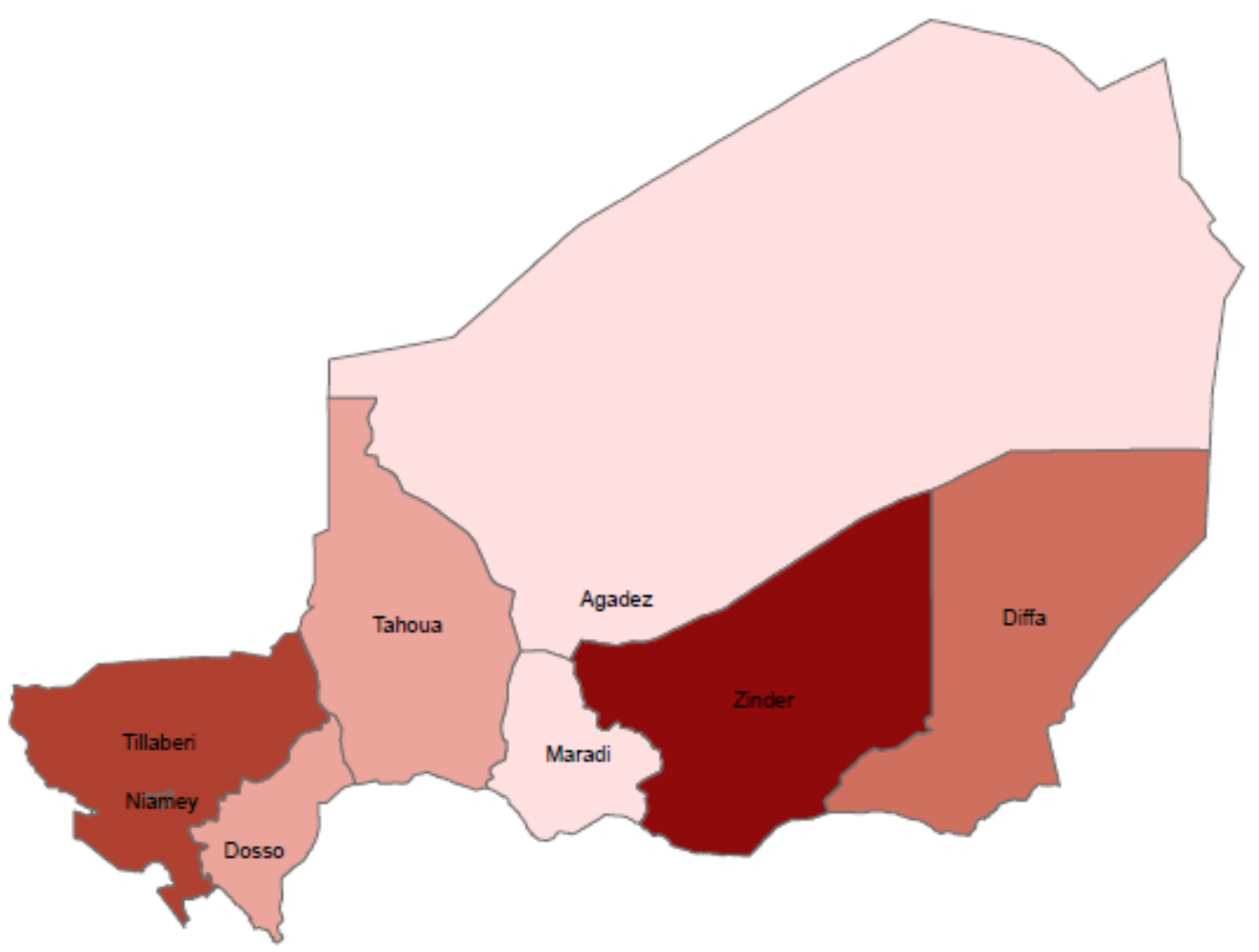

Pourcentage des filles (10-14) non scolarisées et ne vivant avec aucun des parents

\begin{tabular}{|l|l|}
\hline & $6.7-7.3$ \\
$\square$ & $7.4-12.5$ \\
$12.6-14.4$ \\
$14.5-14.9$ \\
$15.0-16.5$
\end{tabular}

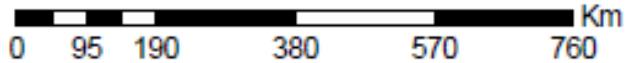




\section{Effectuer une différentiation des services en direction des jeunes déscolarisés}

L'augmentation du taux d'élèves scolarisés ces 20 ou 30 dernières années dans les pays en développement signifie que de plus en plus de jeunes sont inscrits à l'école pendant leur adolescence, et que beaucoup sont encore scolarisés au-delà de l'âge de 15 ans. En dépit de cette tendance, on note encore de très grandes différences de taux de scolarisation notamment en fonction du sexe, du lieu de résidence (en zone rurale ou en zone urbaine) et de la situation matrimoniale. Les interventions à effectuer auprès des jeunes qui ne sont plus scolarisés sont complètement différentes de celles à effectuer auprès de ceux qui le sont encore ; les premiers, et parmi eux en particulier les jeunes filles, ont en effet de fortes chances d'être déjà mariés, d'avoir des enfants et d'être actifs sexuellement. Ces situations s'accompagnent de risques en matière de santé de la procréation et il conviendra de s'occuper de ces risques en dehors de l'environnement scolaire et de façon à ce que les services soient accessibles aux jeunes à risque qui peuvent avoir une mobilité limitée du fait des responsabilités très élevées qu'ils exercent au sein de la famille ou du fait de problèmes de sécurité au sein de leur communauté.

Tableau 7 : Scolarisation des 10-17 ans

\begin{tabular}{|c|c|c|c|c|}
\hline \multicolumn{4}{|c|}{ Pourcentage des adolescents de 10 à 17 ans } \\
actuellement scolarisés \\
\hline \multirow{2}{*}{ Age } & \multicolumn{2}{|c|}{ Urbain } & \multicolumn{2}{c|}{ Rural } \\
\cline { 2 - 5 } & Filles & Garçons & Filles & Garçons \\
\hline 10 & 65.8 & 71.1 & 24.8 & 41.6 \\
\hline 11 & 77.4 & 77.4 & 26.7 & 42.1 \\
\hline 12 & 61.8 & 65.2 & 24.5 & 39.8 \\
\hline 13 & 50.9 & 66.3 & 18.3 & 38.3 \\
\hline 14 & 45.7 & 61.3 & 15.5 & 28.4 \\
\hline 15 & 45.2 & 54.6 & 4.5 & 18.1 \\
\hline 16 & 29.8 & 47.3 & 2.1 & 16.0 \\
\hline 17 & 37.8 & 46.1 & 3.7 & 5.8 \\
\hline
\end{tabular}

Lire la première colonne des données comme suit : « Le pourcentage des filles de 10-17 ans qui sont scolarisées et qui habitaient dans des zones urbaines pendant la collecte des données. » 


\section{Graphique 7 : Pourcentage des adolescents de 10-17 ans actuellement scolarisés}

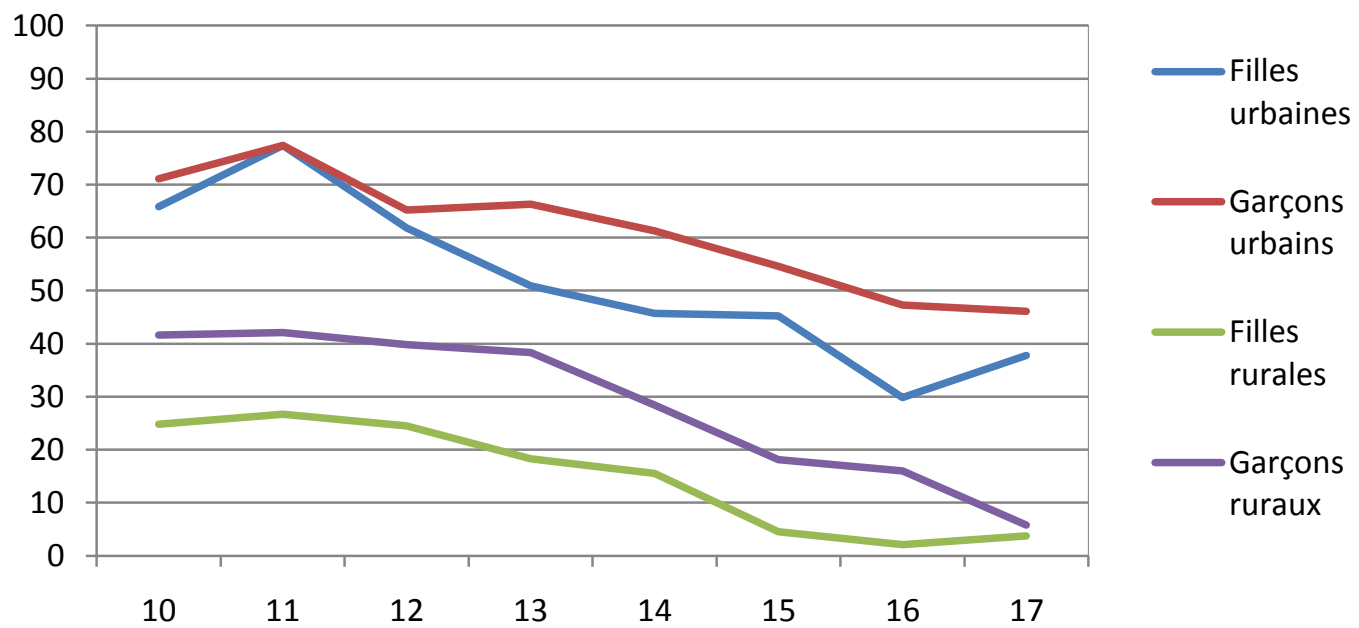

Tableau 8 : Enfants en âge d'aller à l'écolea mais non-scolarisés

\begin{tabular}{|c|c|c|c|}
\hline \multicolumn{3}{|c|}{ Pourcentage des 7-19 ans non-scolarisés ${ }^{\mathrm{b}}$} \\
\hline Région & Filles & Garçons & $\begin{array}{c}\text { Rapport } \\
\text { Filles/garçons }\end{array}$ \\
\hline Agadez & 54.3 & 50.4 & 1.1 \\
\hline Diffa & 78.3 & 71.6 & 1.1 \\
\hline Dosso & 70.2 & 52.3 & 1.3 \\
\hline Maradi & 80.4 & 59.7 & 1.3 \\
\hline Tahoua & 82.3 & 63.9 & 1.3 \\
\hline Tillabéri & 77.7 & 67.7 & 1.1 \\
\hline Zinder & 78.4 & 71.4 & 1.1 \\
\hline Niamey & 42.9 & 33.2 & 1.3 \\
\hline Urbaine & $\mathbf{4 6 . 5}$ & $\mathbf{3 7 . 4}$ & $\mathbf{1 . 2}$ \\
\hline Rurale & $\mathbf{8 1 . 4}$ & $\mathbf{6 6 . 8}$ & $\mathbf{1 . 2}$ \\
\hline $\begin{array}{c}\text { Niveau } \\
\text { national }\end{array}$ & $\mathbf{7 5 . 0}$ & $\mathbf{6 1 . 2}$ & $\mathbf{1 . 2}$ \\
\hline
\end{tabular}

Lire la première colonne des données comme suit : « Le pourcentage des filles de 7-19 ans non-scolarisées. »

a La définition de l'âge de scolarisation par l'UNESCO.

${ }^{b}$ Non-scolarisés au moment de l'enquête ou pendant l'année de l'enquête. 
Carte 2 : Filles en âge d'aller à l'école mais non scolarisées (en \%)

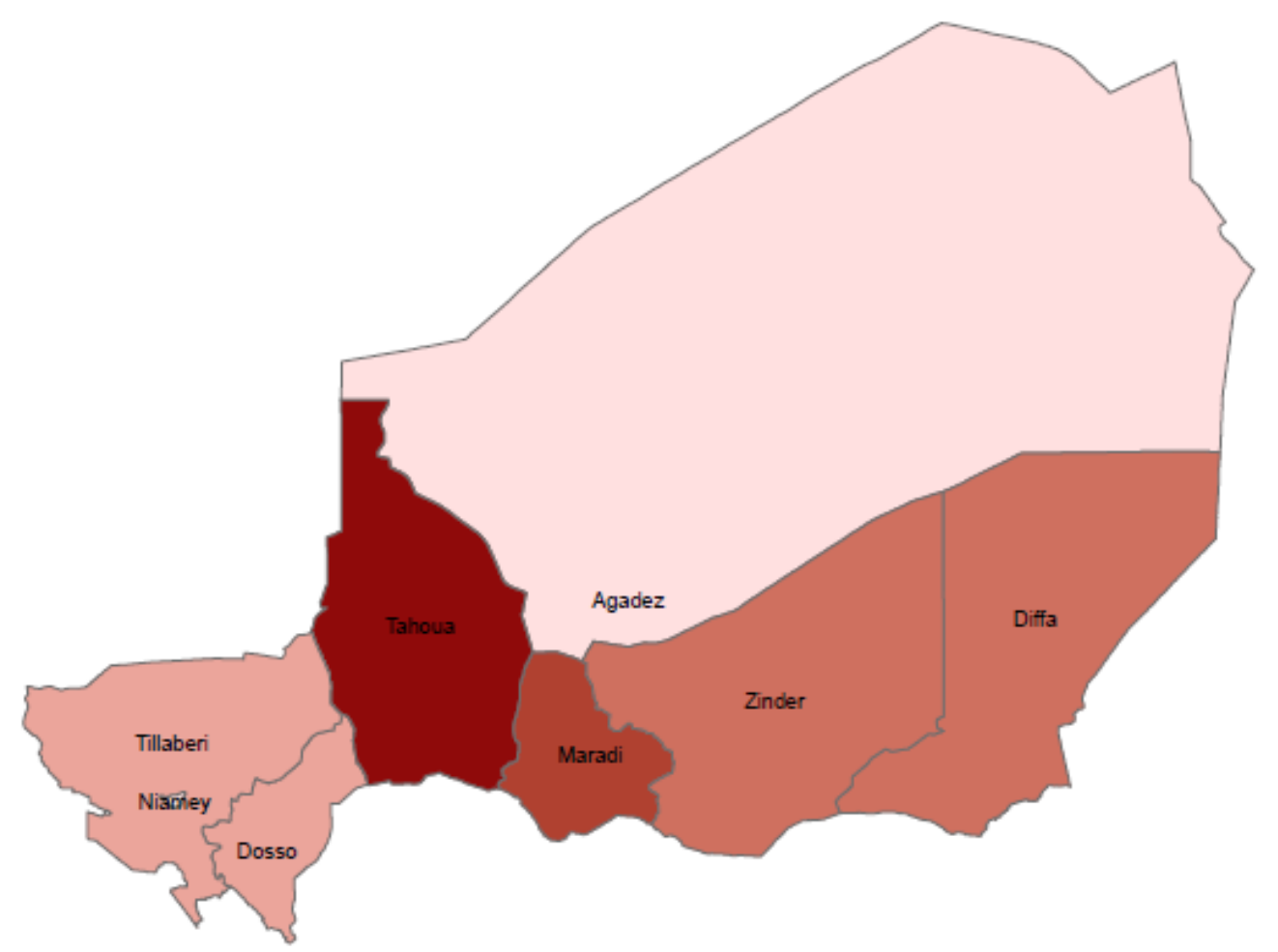

Pourcentage des filles en âge d'aller à l'école mais non scolarisées

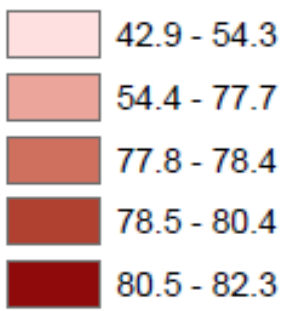

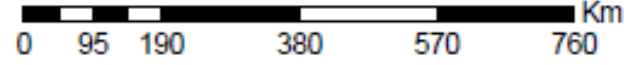


Tableau 9 : Analphabétisme chez les filles

\begin{tabular}{|c|c|c|}
\hline \multicolumn{3}{|c|}{$\begin{array}{c}\text { Pourcentage des filles analphabètes } \\
\text { (incapables de lire une phrase) }\end{array}$} \\
\hline Région & $\begin{array}{c}15-19 \\
\text { ans }\end{array}$ & $\begin{array}{c}20-24 \\
\text { ans }\end{array}$ \\
\hline Agadez & 51.6 & 77.0 \\
\hline Diffa & 79.1 & 87.7 \\
\hline Dosso & 85.5 & 90.4 \\
\hline Maradi & 92.1 & 95.1 \\
\hline Tahoua & 89.2 & 90.8 \\
\hline Tillabéri & 87.8 & 92.9 \\
\hline Zinder & 85.0 & 88.0 \\
\hline Niamey & 48.3 & 49.9 \\
\hline Urbaine & $\mathbf{4 8 . 2}$ & $\mathbf{5 7 . 0}$ \\
\hline Rurale & $\mathbf{9 3 . 2}$ & $\mathbf{9 4 . 7}$ \\
\hline Niveau national & $\mathbf{8 2 . 4}$ & $\mathbf{8 6 . 5}$ \\
\hline
\end{tabular}

Lire la première colonne des données comme suit : « Le pourcentage des filles de 15-19 ans qui étaient incapables de lire une phrase pendant l'enquête. » 
Carte 3 : Analphabétisme chez les filles de 15-19 ans

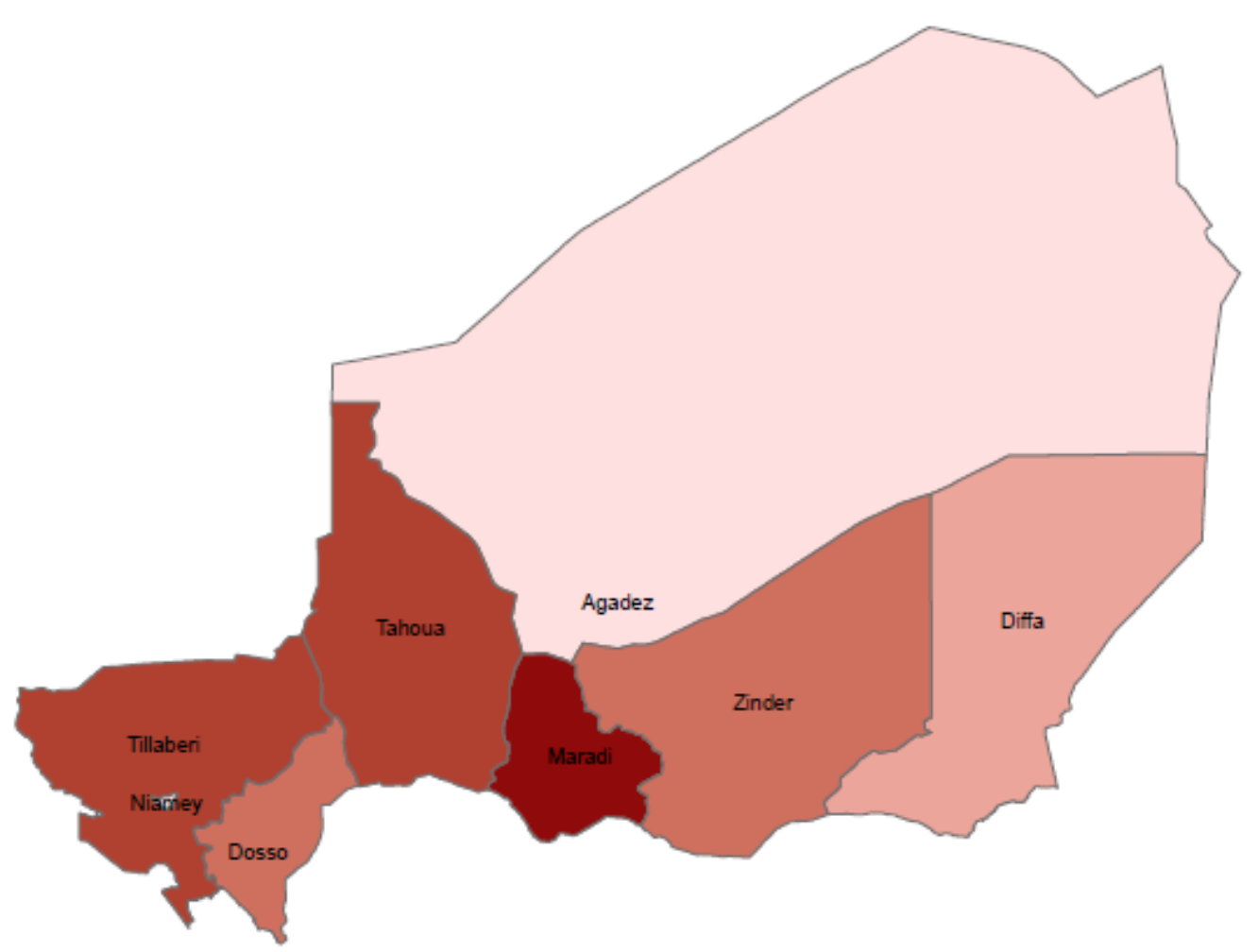

Pourcentage des filles (15-19) qui sont incapables de lire une phrase

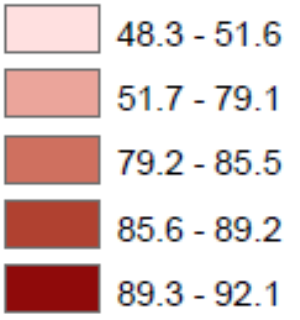

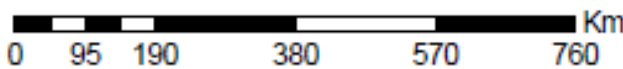




\section{Conséquences d'une scolarisation insuffisante ou non réussie sur la santé de la procréation}

La scolarisation est bénéfique tant pour les jeunes garçons que pour les jeunes filles, et ceci en dehors de tout autre considération, telle que la qualité de l'enseignement par exemple. En ce qui concerne les jeunes filles, elle présente de très grands avantages en matière de santé de la procréation. Les jeunes filles scolarisées au moment de l'adolescence (même si elles ne sont pas inscrites dans la classe qui correspond à leur âge) ont des taux d'activité sexuelle inférieurs à ceux des jeunes filles non scolarisées. Si elles sont déjà actives sexuellement, il est probable qu'elles auront davantage tendance à négocier des rapports protégés et qu'elles auront moins d'enfants. Lorsque les données sont disponibles, il est intéressant de noter à quel âge les jeunes filles commencent en général à l'école (souvent vers 6 ans ou un peu plus tard) et à quel âge certains groupes d'adolescents commencent à quitter l'école. La trajectoire est très souvent très différente selon qu'il s'agit d'un jeune garçon ou d'une jeune fille et que l'on se trouve en zone urbaine ou en zone rurale. Les jeunes filles des zones rurales ont typiquement les taux de scolarisation les plus bas et les taux d'abandon sont plus importants et plus précoces que chez les jeunes garçons ou chez les jeunes filles des zones urbaines. Si les données existent, les décideurs et les concepteurs de programmes à destination des jeunes doivent s'intéresser à au moins quatre groupes :

- les jeunes filles qui n’ont jamais été scolarisées

- les jeunes filles qui ne sont pas scolarisées actuellement mais qui l'ont été

- les jeunes filles scolarisées qui sont dans la classe qui correspond à leur âge

- les jeunes filles qui sont en retard dans leur scolarisation

Il existe une corrélation très forte entre une scolarisation insuffisante (ainsi que d'autres facteurs liés à l'éducation comme l'alphabétisation par exemple) et un mariage précoce. Dans de nombreux cas, le niveau d'éducation a également un impact significatif sur l'âge auquel la jeune fille a son premier enfant, sur son taux de fécondité et sur son manque d'accès à des moyens de contraception souhaités. 
Tableau 10 : Analphabétisme et mariage avant l'âge de 15 ans

\begin{tabular}{|c|c|c|}
\hline \multicolumn{3}{|c|}{$\begin{array}{c}\text { Pourcentage des filles de 15-24 ans qui sont } \\
\text { analphabètes (incapables de lire une phrase) }\end{array}$} \\
\hline Région & $\begin{array}{c}\text { Parmi celles } \\
\text { mariées } \\
\text { avant l'âge } \\
\text { de 15 ans }\end{array}$ & $\begin{array}{c}\text { Parmi } \\
\text { celles qui } \\
\text { n'ont } \\
\text { jamais été } \\
\text { mariées }\end{array}$ \\
\hline Agadez & 90.8 & 47.3 \\
\hline Diffa & 96.2 & 53.7 \\
\hline Dosso & 98.0 & 68.5 \\
\hline Maradi & 98.8 & $(60.4)$ \\
\hline Tahoua & 94.2 & 76.6 \\
\hline Tillabéri & 93.7 & 81.6 \\
\hline Zinder & 94.7 & 47.3 \\
\hline Niamey & $(87.3)$ & 39.1 \\
\hline Urbaine & $\mathbf{8 3 . 3}$ & $\mathbf{3 7 . 4}$ \\
\hline Rurale & $\mathbf{9 6 . 8}$ & $\mathbf{8 3 . 1}$ \\
\hline Niveau national & $\mathbf{9 5 . 7}$ & $\mathbf{5 9 . 6}$ \\
\hline
\end{tabular}

Lire la première colonne des données comme suit : « Le pourcentage des filles de 15-24 ans qui se sont mariées avant l'âge de 15 ans et qui étaient incapables de lire une phrase pendant l'enquête. »

Les chiffres entre parenthèses sont basés sur 25-50 cas non pondérés. 


\section{Graphique 8 : Pourcentage des filles de 15-24 ans qui sont analphabètes, par statut matrimonial}

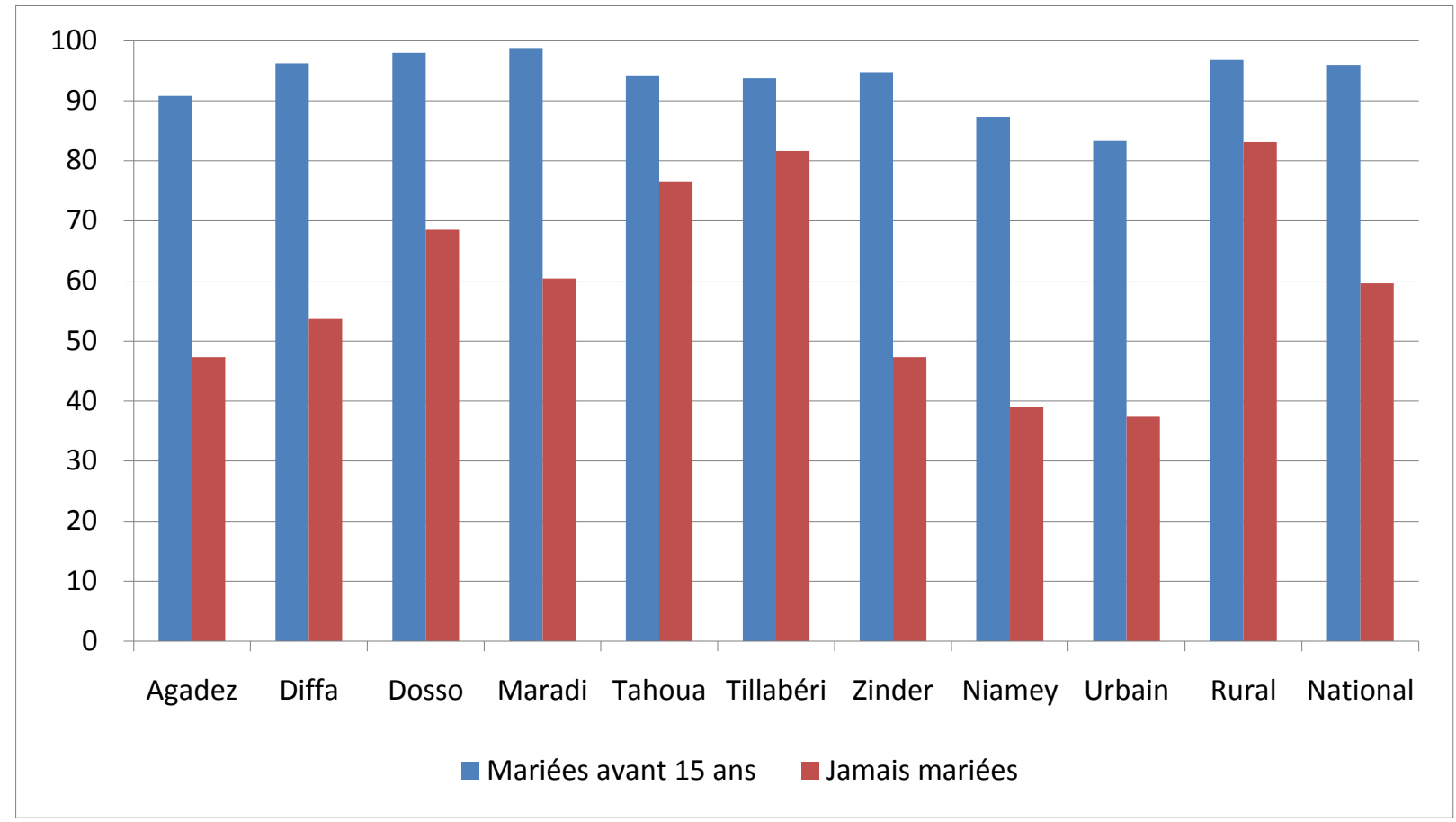

Tableau 11 : Scolarisation en fonction du statut matrimonial et de la maternité

\begin{tabular}{|c|c|c|c|c|}
\hline \multicolumn{5}{|c|}{ Pourcentage des filles de 15-19 ans scolarisées en fonction du } \\
statut matrimonial et de la maternité \\
\hline Région & $\begin{array}{c}\text { Déjà } \\
\text { mariées, } \\
\text { avec } \\
\text { enfant }\end{array}$ & $\begin{array}{c}\text { Jamais } \\
\text { mariées, } \\
\text { avec } \\
\text { enfant }\end{array}$ & $\begin{array}{c}\text { Déjà } \\
\text { mariées, } \\
\text { sans } \\
\text { enfant }\end{array}$ & $\begin{array}{c}\text { Jamais } \\
\text { mariées, } \\
\text { sans } \\
\text { enfant }\end{array}$ \\
\hline Urbain & $\mathbf{2 . 5}$ & $*$ & $\mathbf{( 6 . 5 )}$ & $\mathbf{5 3 . 0}$ \\
\hline Rurale & $\mathbf{0 . 9}$ & $\mathbf{( 5 . 0 )}$ & $\mathbf{1 1 . 2}$ & $\mathbf{5 5 . 1}$ \\
\hline $\begin{array}{c}\text { Niveau } \\
\text { national }\end{array}$ & $\mathbf{1 . 3}$ & $\mathbf{( 4 . 2 )}$ & $\mathbf{1 0 . 2}$ & $\mathbf{5 4 . 2}$ \\
\hline
\end{tabular}

Lire la première colonne des données comme suit : « Le pourcentage des filles de 15-19 ans mariées et ayant un enfant et qui étaient scolarisées au moment de l'enquête. »

Les chiffres entre parenthèses sont basés sur 25-50 cas non pondérés.

* Indique que le chiffre est basé sur moins de 25 cas non pondérés et a été supprimé.

a Scolarisées pendant l'année scolaire.

${ }^{\mathrm{b}}$ Un enfant qui vit dans le même foyer que sa mère. 


\section{C-Contexte de l'activité sexuelle : le mariage précoce, les normes de genre et la santé de la procréation}

Pour pouvoir analyser les résultats en matière de santé de la procréation chez les adolescents, il est indispensable de comprendre le contexte de l'initiation à la sexualité chez les jeunes et de savoir que les jeunes filles sont particulièrement vulnérables et ont davantage tendance à avoir des rapports sexuels forcés (tant au sein du mariage qu'en dehors). Les jeunes gens et les jeunes filles découvrent la sexualité dans un contexte de normes de genre très fortes et dans un environnement où la pression des parents ou des pairs s'exerce pour qu'ils aient (ou n'aient pas) des relations sexuelles et des enfants. Ces normes de genre peuvent autoriser le recours à la force à l'égard des femmes ainsi que d'autres pratiques révélatrices du déséquilibre du rapport de forces dans les relations hommes-femmes. Ces normes peuvent jouer un rôle majeur dans l'accès à l'information dont disposent les jeunes filles, notamment en matière de santé reproductive et en ce qui concerne leurs droits. En outre, ces normes peuvent expliquer que les jeunes filles soient soumises à des relations sexuelles non souhaitées et non protégées, et ceci au sein du mariage ou en dehors de celui-ci. Cette section a pour but de présenter des données relatives aux différents contextes dans lesquels les adolescents ont des relations sexuelles ainsi que des données sur une sélection d'indicateurs de la santé de la procréation.

\section{Le mariage précoce et les risques qui lui sont associés}

Le mariage précoce est encore très pratiqué dans de nombreux pays, et est extrêmement fréquent dans certaines régions bien spécifiques. Certains invoquent des "normes culturelles » pour justifier le mariage avant 18 ans et parfois même avant 15 ans, bien qu'il soit interdit dans beaucoup de pays. La plupart des pays ont signé la Convention sur l'élimination de toutes les formes de discrimination à l'égard des femmes et la Convention relative aux droits de l'enfant qui précisent que les filles et les garçons ont le droit de profiter pleinement de leur jeunesse jusqu'à l'âge de 18 ans. En dépit de la mise en place de cadres juridiques en leur faveur, les normes culturelles, les forces économiques (telles qu'elles sont perçues par les familles) et les normes de genre limitent l'accès des jeunes et en particulier des jeunes filles - à une information essentielle sur la santé de la procréation et sur la protection de leurs droits. Les relations sexuelles des jeunes ayant atteint l'âge légal de la majorité devraient être voulues, protégées et satisfaisantes. Il est donc important d'intervenir dans de nombreuses régions pour mettre un terme au mariage précoce, aux relations sexuelles à des fins économiques et à l'acceptation de la violence dans les relations hommes-femmes. Il faut de plus mettre tout en œuvre pour que les enfants, au moment de l'adolescence, aient accès à l'information qui leur est nécessaire pour faire des choix conscients et sûrs en matière de santé de la procréation ainsi que pour tout ce qui concerne leur vie.

La persistance du mariage précoce a également une conséquence démographique importante : l'écart entre les générations est très court, ce qui est un facteur essentiel dans la dynamique de croissance de la population. Plusieurs facteurs se conjuguent pour expliquer l'augmentation du nombre de grossesses chez les jeunes filles mariées très jeunes. Le déséquilibre du rapport de forces au sein du couple s'explique par le jeune âge de l'épouse et celui, en général plus élevé, de son époux. Ce jeune âge est aussi révélateur d'un niveau d'éducation assez bas. Pour compenser ses lacunes en matière d'éducation et son manque de compétences, la jeune mariée tente d'assurer son avenir tant au sein du couple que d'un point de vue social en ayant un grand nombre d'enfants. Ceci peut avoir 
pour effet de déséquilibrer encore davantage le rapport de forces entre les conjoints et donc de limiter encore plus le pouvoir de négociation de la jeune épouse pour s'assurer qu'elle est protégée contre les MST et le VIH lors des relations sexuelles. Les normes de genre peuvent être un obstacle à l'utilisation de contraceptifs ou d'autres moyens pour choisir le nombre d'enfants et la fréquence des grossesses.

Tableau 12 : Age des filles lors du premier mariage ou de la première union

\begin{tabular}{|c|c|c|}
\hline \multicolumn{3}{|c|}{$\begin{array}{c}\text { Age lors du premier mariage ou de la } \\
\text { première union pour les filles de 20-24 } \\
\text { ans (en \%) }\end{array}$} \\
\hline Région & $\begin{array}{c}\text { Mariées } \\
\text { avant 15 } \\
\text { ans }\end{array}$ & $\begin{array}{c}\text { Mariées } \\
\text { avant 18 } \\
\text { ans }\end{array}$ \\
\hline Agadez & 19.9 & 48.4 \\
\hline Diffa & 44.1 & 88.7 \\
\hline Dosso & 32.3 & 73.9 \\
\hline Maradi & 43.4 & 86.7 \\
\hline Tahoua & 35.7 & 83.2 \\
\hline Tillabéri & 25.6 & 68.8 \\
\hline Zinder & 58.0 & 88.2 \\
\hline Niamey & 10.1 & 27.5 \\
\hline Urbaine & $\mathbf{1 4 . 3}$ & $\mathbf{4 2 . 1}$ \\
\hline Rurale & $\mathbf{4 2 . 3}$ & $\mathbf{8 3 . 6}$ \\
\hline $\begin{array}{c}\text { Niveau } \\
\text { national }\end{array}$ & $\mathbf{3 6 . 1}$ & $\mathbf{7 4 . 5}$ \\
\hline
\end{tabular}

Lire la première colonne des données comme suit : « Le pourcentage des filles de 20-24 ans mariées avant l'âge de 15 ans. » 
Graphique 9 : Pourcentage des filles de 20-24 ans mariées avant 15 et 18 ans

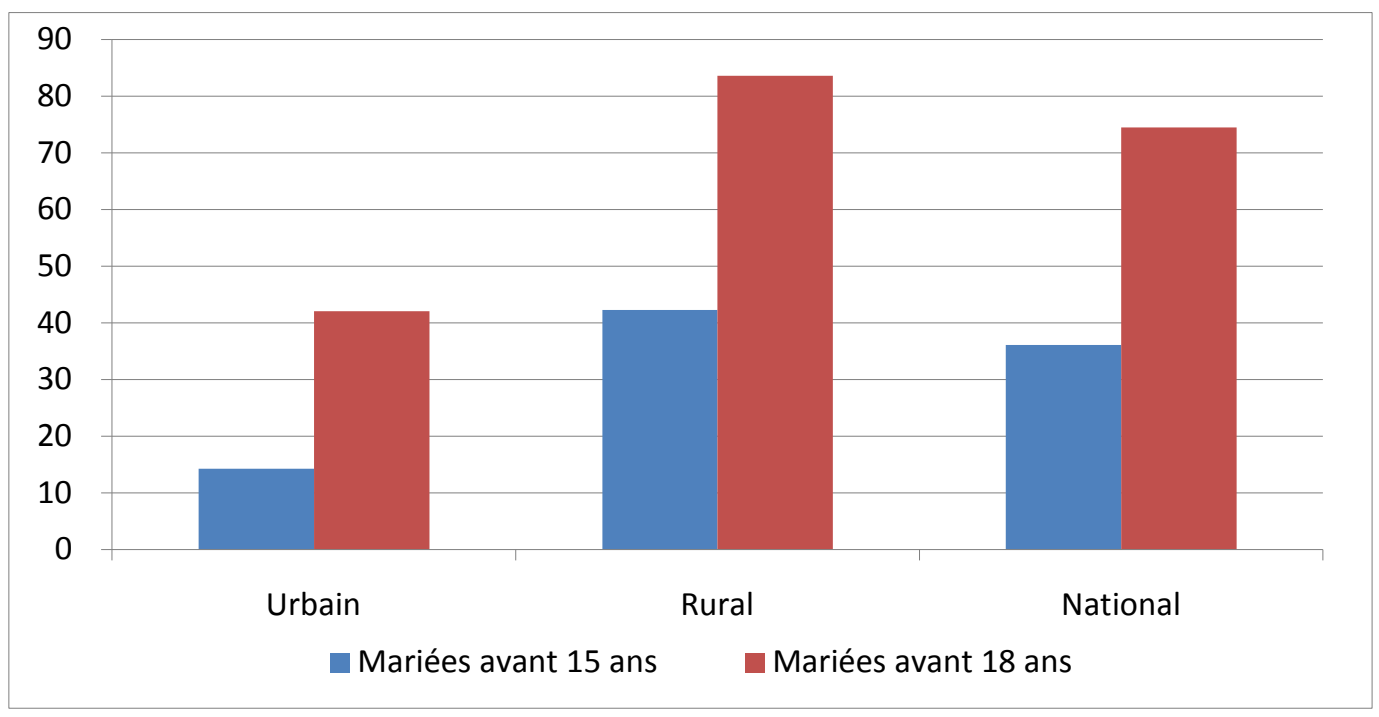


Carte 4 : Mariage précoce chez les filles de 20-24 ans

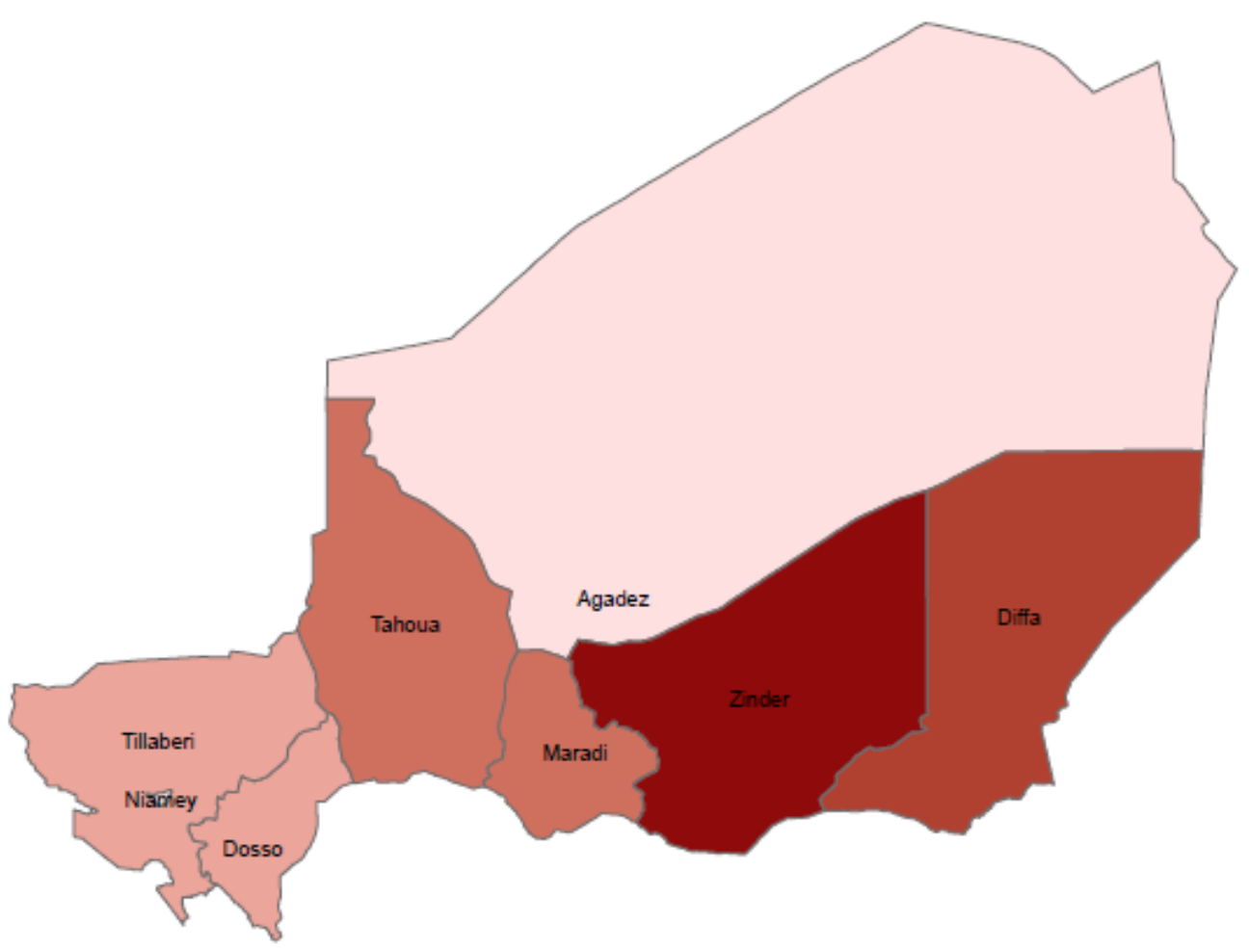

Pourcentage des filles (de 20 à 24 ans) qui se sont mariées avant 15 ans

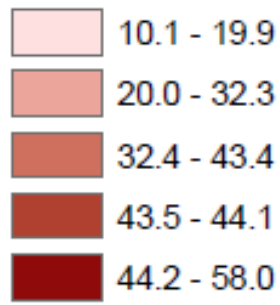

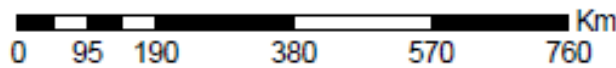




\section{Soutenir les jeunes filles enceintes et les jeunes mères}

Les jeunes mères, qu'elles soient mariées ou non, ont besoin d'un soutien social et médical. Les jeunes filles mariées ont souvent moins de mobilité, un accès réduit à l'information et moins d'autonomie pour prendre des décisions, que les femmes mariées plus âgées. Elles sont souvent isolées de leurs pairs et plus susceptibles d'être victimes de violences liées au genre que les femmes qui se marient plus tard. L'ensemble de ces facteurs peut réduire l'accès qu'ont ces jeunes filles enceintes à l'information, aux services, et à toute forme de soutien. Ce sont les plus jeunes d'entre elles qui présentent le plus grand risque de morbidité et de mortalité maternelle lors d'une première grossesse. Les programmes mis en place en direction des jeunes filles et des couples ayant leur premier enfant commencent à montrer que des interventions ciblées encouragent le recours aux soins prénataux et aux tests de dépistage du VIH (et, dans l'idéal, au partage de l'information avec le partenaire), et favorisent de meilleures pratiques d'allaitement et de meilleurs soins aux enfants.

\section{Tableau 13 : Grossesse et maternité}

\begin{tabular}{|c|c|c|c|}
\hline \multicolumn{4}{|c|}{$\begin{array}{c}\text { Pourcentage des filles de } \\
\text { enceintes ou ayant eu un enfant }{ }^{\mathrm{a}} \text { anjà été }\end{array}$} \\
\hline Région & $\begin{array}{c}\text { Mariées } \\
\text { ou l'ayant } \\
\text { étéb }\end{array}$ & $\begin{array}{c}\text { Jamais } \\
\text { mariées }\end{array}$ & $\begin{array}{c}\text { Total des } \\
\text { filles de 15- } \\
19 \text { ans }\end{array}$ \\
\hline Agadez & 77.9 & 1.6 & 20.3 \\
\hline Diffa & 79.7 & 2.6 & 56.3 \\
\hline Dosso & 61.4 & 0.0 & 34.4 \\
\hline Maradi & 67.6 & $(0.0)$ & 57.6 \\
\hline Tahoua & 65.4 & 0.8 & 43.0 \\
\hline Tillabéri & 58.0 & 1.8 & 32.9 \\
\hline Zinder & 66.5 & 0.0 & 49.2 \\
\hline Niamey & 76.0 & 1.1 & 14.6 \\
\hline Urbaine & $\mathbf{7 7 . 1}$ & $\mathbf{1 . 4}$ & $\mathbf{2 0 . 5}$ \\
\hline Rurale & $\mathbf{6 4 . 1}$ & $\mathbf{0 . 4}$ & $\mathbf{4 6 . 2}$ \\
\hline $\begin{array}{c}\text { Niveau } \\
\text { national }\end{array}$ & $\mathbf{6 5 . 4}$ & $\mathbf{0 . 8}$ & $\mathbf{4 0 . 0}$ \\
\hline
\end{tabular}

Lire la première colonne des données comme suit : « Le pourcentage des filles de 15-19 ans déjà mariées ou en union ayant déjà été enceintes ou ayant eu un enfant. » Les chiffres entre parenthèses sont basés sur 25-50 cas non pondérés.

a Inclut des femmes qui ont eu un enfant, sont enceintes actuellement, ont eu une fausse couche, ou ayant perdu un enfant pour d'autres raisons.

b Inclut celles qui sont mariées, en union, séparées, divorcées, ou veuves. 


\section{Graphique 10 : Pourcentage des filles de 15-19 ans ayant déjà été enceintes ou ayant eu un enfant}

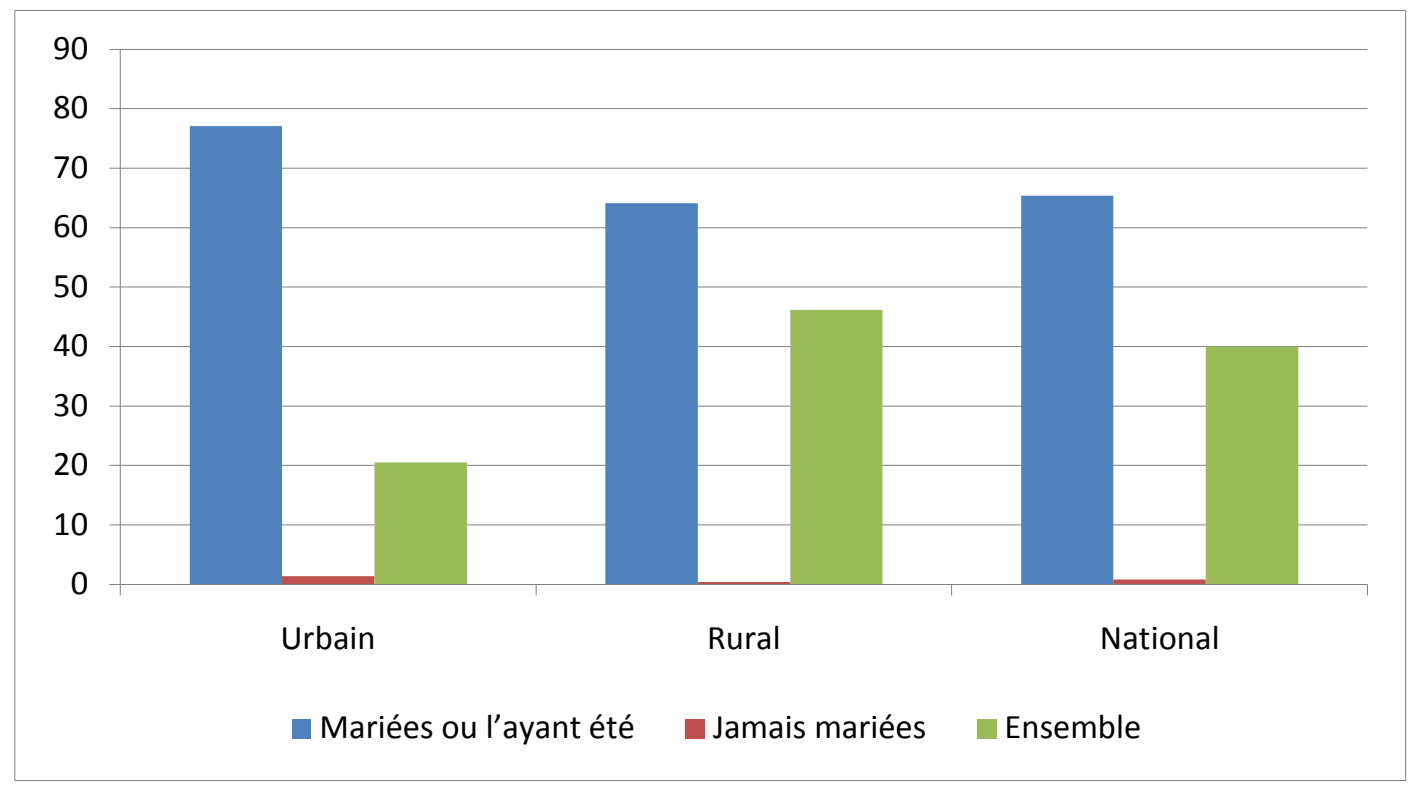

\section{La question de l'activité sexuelle tant au sein du mariage qu'en dehors}

Il est essentiel de bien comprendre qu'il existe deux situations bien différentes dans lesquelles les jeunes gens ont des relations sexuelles : au sein du mariage ou en dehors du mariage. Bien qu'une part de plus en plus importante des relations sexuelles - y compris l'initiation sexuelle - ait lieu en dehors du mariage, il est vrai que dans la plupart des pays l'activité sexuelle de la majorité des jeunes adolescentes a lieu au sein du couple. Or la sexualité entre époux ne garantit pas des rapports sans risque, souhaités ou satisfaisants. Dans certains cas, les jeunes filles mariées ont un risque plus élevé de contracter une maladie sexuellement transmissible ou le VIH que celles qui sont sexuellement actives mais ne sont pas mariées. Qu'elles soient mariées ou non, ces jeunes adolescentes ont besoin d'être informées et de bénéficier d'un soutien pour pouvoir prendre des décisions réfléchies ; il leur faut aussi avoir accès à des services et une technologie médicale afin de conserver une bonne santé sexuelle et de la procréation. Il est vital de bien comprendre la nature de la relation entre les partenaires et le contexte des relations sexuelles si l'on veut mettre en place des programmes efficaces. Bien que leurs besoins se recoupent, les jeunes filles ont également des besoins bien spécifiques selon qu'elles soient mariées ou non pour maintenir une bonne santé sexuelle et de la procréation. 
Tableau 14 : Activité sexuelle chez les filles de 15-19 ans

\begin{tabular}{|c|c|c|c|c|c|c|}
\hline \multicolumn{7}{|c|}{ Activité sexuelle chez les filles de 15-19 ans (en \%) } \\
\hline \multirow{2}{*}{ Région } & \multicolumn{2}{|c|}{$\begin{array}{c}\text { Déjà eu un } \\
\text { rapport sexuel }\end{array}$} & \multicolumn{3}{|c|}{ Active sexuellement ${ }^{\mathrm{a}}$} & \multirow{2}{*}{$\begin{array}{l}\text { Filles de 15- } \\
19 \text { ans } \\
\text { actives } \\
\text { sexuellement } \\
\text { et mariées } \\
\text { (en \%) }\end{array}$} \\
\hline & $\begin{array}{l}\text { Jamais } \\
\text { mariées }\end{array}$ & Total & $\begin{array}{l}\text { Actuellement } \\
\text { mariées }\end{array}$ & $\begin{array}{l}\text { Jamais } \\
\text { mariées }\end{array}$ & Total & \\
\hline Agadez & 4.0 & 27.6 & (83.4) & 1.2 & 18.4 & $(95.0)$ \\
\hline Diffa & 0.0 & 69.7 & 82.0 & 0.0 & 55.8 & 98.7 \\
\hline Dosso & 0.0 & 54.4 & 48.7 & 0.0 & 26.7 & 100.0 \\
\hline Maradi & $(0.0)$ & 85.2 & 72.5 & $(0.0)$ & 61.3 & 100.0 \\
\hline Tahoua & 0.8 & 64.6 & 52.8 & 0.0 & 34.2 & 99.1 \\
\hline Tillabéri & 2.0 & 56.1 & 58.2 & 0.0 & 31.2 & 100.0 \\
\hline Zinder & 0.0 & 73.7 & 65.1 & 0.0 & 45.4 & 100.0 \\
\hline Niamey & 1.7 & 19.3 & $(50.8)$ & 0.0 & 9.0 & (92.7) \\
\hline Urbaine & 2.0 & 26.6 & 65.4 & 0.2 & 15.9 & 95.7 \\
\hline Rurale & 0.3 & 71.3 & 60.4 & 0.0 & 42.4 & 100.0 \\
\hline $\begin{array}{c}\text { Niveau } \\
\text { national }\end{array}$ & 1.1 & 60.5 & 60.9 & 0.1 & 36.1 & 99.5 \\
\hline
\end{tabular}

Lire la première colonne des données comme suit : « Le pourcentage des filles de 15-19 qui ne sont jamais mariées et qui ont déjà eu un rapport sexuel. »

Les chiffres entre parenthèses sont basés sur 25-50 cas non pondérés.

${ }^{a}$ Se rapporte aux filles qui ont eu des rapports sexuels durant les 4 semaines précédant l'enquête. 
Tableau 15 : Initiation sexuelle chez les filles de 20-24 ans

\begin{tabular}{|c|c|c|c|}
\hline \multicolumn{3}{|c|}{ Initiation sexuelle chez les filles ayant actuellement entre 20 et 24 ans } \\
(en \%) \\
\hline Région & $\begin{array}{c}\text { Ayant eu un } \\
\text { rapport sexuel } \\
\text { avant 15 ans }\end{array}$ & $\begin{array}{c}\text { Ayant eu un } \\
\text { rapport sexuel } \\
\text { avant 18 ans }\end{array}$ & $\begin{array}{c}\text { Premier } \\
\text { rapport sexuel } \\
\text { au mariage }\end{array}$ \\
\hline Agadez & 16.8 & 48.5 & 42.2 \\
\hline Diffa & 43.3 & 88.7 & 67.7 \\
\hline Dosso & 23.7 & 73.8 & 30.2 \\
\hline Maradi & 42.3 & 86.4 & 84.6 \\
\hline Tahoua & 34.7 & 83.6 & 33.2 \\
\hline Tillabéri & 22.8 & 68.6 & 59.3 \\
\hline Zinder & 53.2 & 88.1 & 74.6 \\
\hline Niamey & 11.2 & 28.6 & 17.7 \\
\hline Urbaine & $\mathbf{1 4 . 4}$ & $\mathbf{4 2 . 5}$ & $\mathbf{3 1 . 3}$ \\
\hline Rurale & $\mathbf{3 8 . 6}$ & $\mathbf{8 3 . 6}$ & $\mathbf{5 8 . 7}$ \\
\hline Niveau national & $\mathbf{3 3 . 3}$ & $\mathbf{7 4 . 6}$ & $\mathbf{5 2 . 7}$ \\
\hline
\end{tabular}

Lire la premiere colonne des données comme suit : « Le pourcentage des filles de 20-24 ans qui ont eu un rapport sexuel avant l'âge de 15. » 


\section{Graphique 11 : Initiation sexuelle chez les filles de 20-24 ans, par région} (en \%)

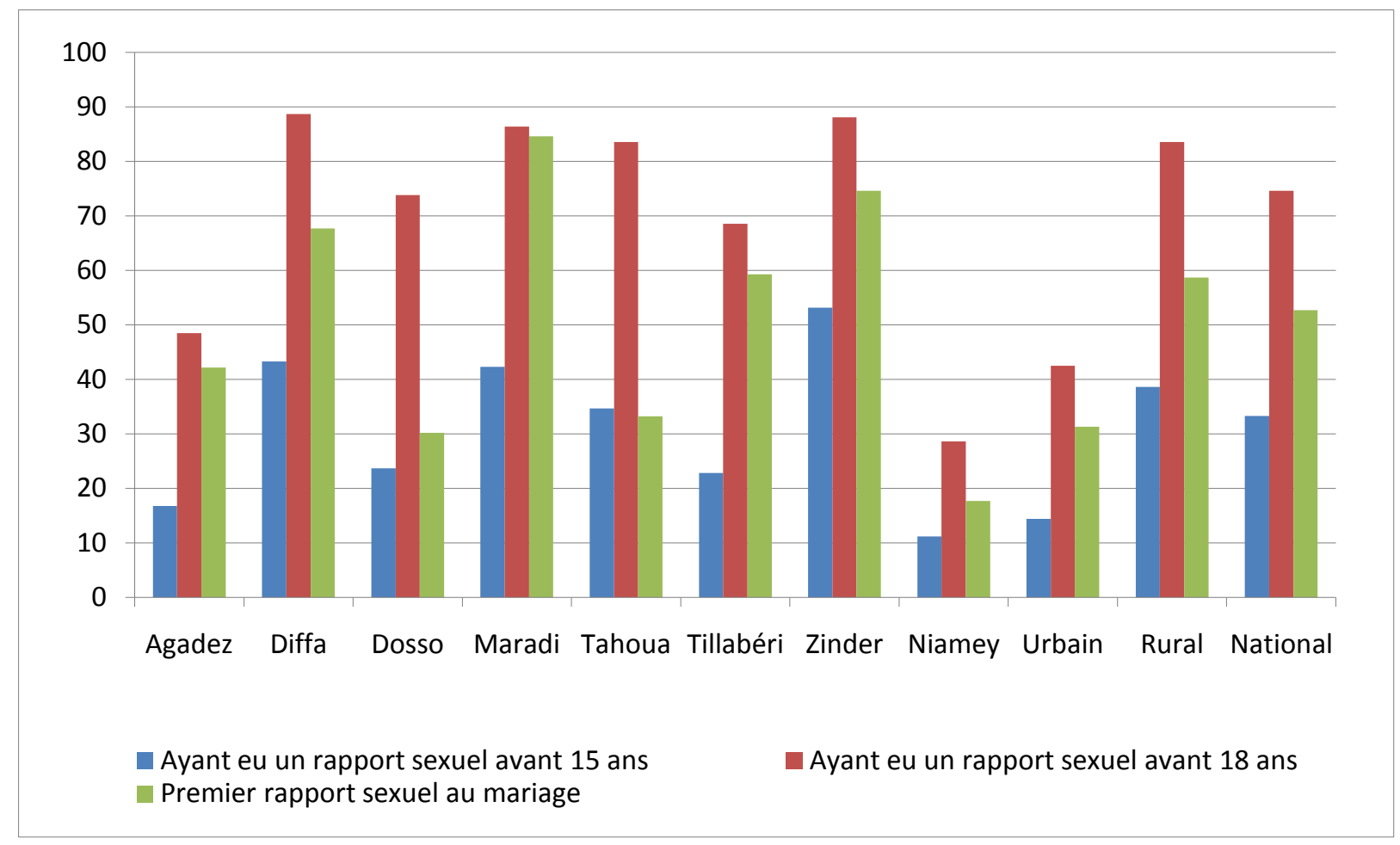

\section{La différence d'âge entre les jeunes adolescentes et leurs partenaires}

Dans les pays en développement, plus une jeune fille est mariée jeune, plus, en général, la différence d'âge avec son mari est importante. En moyenne, les maris des jeunes épouses sont plus âgés que les partenaires sexuels des jeunes filles non mariées. La différence d'âge a deux dimensions importantes :

- L'âge auquel la jeune fille a des rapports sexuels, et son lien avec sa maturité physique et émotionnelle

- L'âge de son partenaire, et son lien avec son expérience sexuelle et par conséquent avec son exposition aux MST ou au VIH.

L'importante différence d'âge entre ces jeunes filles et leurs partenaires ou époux les place dans une situation défavorable et réduit leurs chances d'échapper à la violence ou de négocier des rapports sexuels sûrs. Qu'elles soient mariées ou non, cette différence d'âge a un impact sur leurs capacités à négocier des relations sexuelles non contraintes, sûres et protégées. La recherche effectuée sur cette question montre que les mariages entre jeunes filles et hommes plus âgés sont moins équitables ${ }^{8}$.

\footnotetext{
${ }^{8}$ Clark, Shelley, Judith Bruce, and Annie Dude. 2006. "Protecting Young Women from HIV/AIDS: The Case Against Child and Adolescent Marriage," International Family Planning Perspectives, 32(2): 79 88.
} 
Tableau 16 : Rapports sexuels forcés chez les filles de 15-24 ans

\begin{tabular}{|c|c|}
\hline \multicolumn{2}{|c|}{$\begin{array}{c}\text { Pourcentage des filles de } 15-24 \\
\text { dont le premier rapport sexuel a } \\
\text { été forcé }\end{array}$} \\
\hline Région & $\begin{array}{c}\text { Premier rapport } \\
\text { sexuel forcé }\end{array}$ \\
\hline Agadez & 20.8 \\
\hline Diffa & 40.4 \\
\hline Dosso & 9.6 \\
\hline Maradi & 6.1 \\
\hline Tahoua & 20.8 \\
\hline Tillabéri & 6.6 \\
\hline Zinder & 16.3 \\
\hline Niamey & 23.0 \\
\hline Urbaine & $\mathbf{2 1 . 2}$ \\
\hline Rurale & $\mathbf{1 2 . 2}$ \\
\hline National & $\mathbf{1 3 . 5}$ \\
\hline
\end{tabular}

Lire la première colonne des données comme suit : « Le pourcentage des filles de 15-24 dont le premier rapport sexuel a été forcé. »

Graphique 12 : Pourcentage des filles de 15-24 ans dont le premier rapport sexuel a été forcé

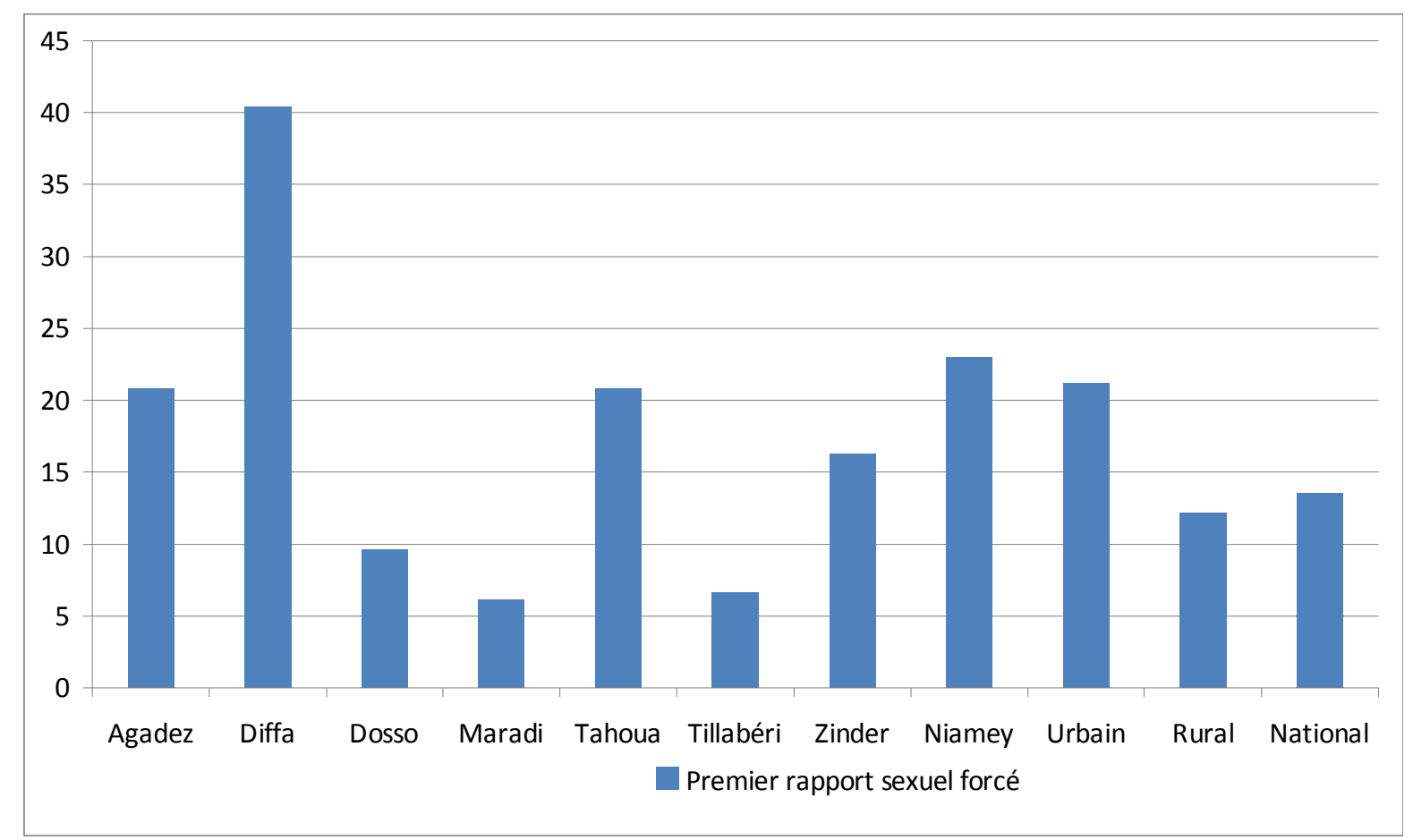




\section{Les normes de genre et la violence conjugale}

Toute relation sexuelle a lieu dans un contexte social. Les normes de genre ont une incidence sur la façon dont les hommes et les femmes perçoivent l'acceptabilité du recours à la violence pour exercer un contrôle sur la mobilité des jeunes filles et des femmes, sur leur pouvoir décisionnel et sur leurs relations sociales, ainsi que sur les conditions dans lesquelles se déroule le rapport sexuel, à quel moment il a lieu et les précautions employées. Très souvent, les garçons et les filles internalisent ces normes à un très jeune âge, ce qui conduit à une inégalité en matière de relations sexuelles. Il y a une corrélation entre des normes de genre injustes et la perception de la violence. Ainsi, une femme qui considère qu'il est légitime pour l'homme d'avoir recours à la violence dans certaines situations aura davantage tendance à déclarer qu'il lui est arrivé d'avoir subi des violences ${ }^{9}$. De plus, il est attesté que la violence envers les femmes a des conséquences néfastes, et induit notamment des grossesses non désirées et des infections à VIH. Les données sur le degré d'acceptabilité de la violence, quand elles existent, peuvent donc donner des indications importantes dont il faut tenir compte si l'on veut élaborer des programmes de santé sexuelle et de la procréation efficaces.

Tableau 17 : Différence d'âge entre les partenaires lors du premier rapport sexuel

\begin{tabular}{|c|c|c|c|}
\hline \multicolumn{4}{|c|}{$\begin{array}{c}\text { Différence d'âge entre la fille et son premier partenaire sexuel, } \\
\text { parmi les filles âgées actuellement de } 15 \text { à } 24 \text { ans (en \%) }\end{array}$} \\
\hline \multirow{2}{*}{ Région } & $\begin{array}{c}\text { Partenaire } \\
\text { plus jeune ou } \\
\text { du même âge }\end{array}$ & $\begin{array}{c}\text { Partenaire } \\
\text { ayant moins } \\
\text { de } 10 \text { ans de } \\
\text { plus }\end{array}$ & $\begin{array}{c}\text { Partenaire } \\
\text { ayant plus de } \\
10 \text { ans de plus }\end{array}$ \\
\hline Urbain & $\mathbf{4 . 2}$ & $\mathbf{3 6 . 4}$ & $\mathbf{5 9 . 4}$ \\
\hline Rural & $\mathbf{7 . 6}$ & $\mathbf{5 5 . 0}$ & $\mathbf{3 7 . 5}$ \\
\hline National & $\mathbf{7 . 1}$ & $\mathbf{5 2 . 6}$ & $\mathbf{4 0 . 3}$ \\
\hline
\end{tabular}

Lire la première colonne des données comme suit : « Le pourcentage des filles de 15-24 ans dont le premier partenaire sexuel était du même âge ou plus jeune qu'elle. »

\footnotetext{
${ }^{9}$ UN Millennium Project. 2006. "Public Choices, Private Decisions: Sexual and Reproductive Health and the Millennium Development Goals." New York: UN Millennium Project
} 
Table 18 : Différence d'âge entre les partenaires actuels

\begin{tabular}{|c|c|c|c|}
\hline \multicolumn{5}{|c|}{$\begin{array}{c}\text { Différence d'âge entre la fille et son mari ou son } \\
\text { partenaire actuel chez les filles de 15-24 ans (en \%) }\end{array}$} \\
\hline Région & $\begin{array}{c}\text { Partenaire } \\
\text { plus jeune ou } \\
\text { du même âge }\end{array}$ & $\begin{array}{c}\text { Partenaire } \\
\text { ayant moins } \\
\text { de } 10 \text { ans } \\
\text { de plus }\end{array}$ & $\begin{array}{c}\text { Partenaire } \\
\text { ayant plus } \\
\text { de 10 ans de } \\
\text { plus }\end{array}$ \\
\hline Agadez & 0.0 & 34.0 & 66.1 \\
\hline Diffa & 0.0 & 52.6 & 47.4 \\
\hline Dosso & 0.0 & 64.8 & 35.2 \\
\hline Maradi & 0.2 & 53.5 & 46.3 \\
\hline Tahoua & 0.3 & 42.0 & 57.6 \\
\hline Tillabéri & 0.0 & 58.5 & 41.5 \\
\hline Zinder & 0.5 & 54.8 & 44.7 \\
\hline Niamey & 0.0 & 34.7 & 65.3 \\
\hline Urbain & $\mathbf{0 . 0}$ & $\mathbf{3 5 . 1}$ & $\mathbf{6 5 . 0}$ \\
\hline Rurale & $\mathbf{0 . 2}$ & $\mathbf{5 5 . 0}$ & $\mathbf{4 4 . 7}$ \\
\hline National & $\mathbf{0 . 2}$ & $\mathbf{5 2 . 5}$ & $\mathbf{4 7 . 3}$ \\
\hline
\end{tabular}

Lire la première colonne des données comme suit : «Le pourcentage des filles de 15-24 ans dont le partenaire sexuel actuel est du même âge ou plus jeune qu'elle. » 


\section{Tableau 19A : Attitude des filles vis-à-vis de la violence conjugale}

\begin{tabular}{|c|c|c|c|}
\hline \multicolumn{4}{|c|}{$\begin{array}{l}\text { Pourcentage des filles de 15-24 ans qui considèrent que la } \\
\text { violence est acceptable quelles que soient les circonstances }\end{array}$} \\
\hline \multirow{2}{*}{ Région } & $\begin{array}{c}\text { Ayant } \\
\text { déjà été } \\
\text { mariées }\end{array}$ & $\begin{array}{c}\text { Jamais } \\
\text { mariées }\end{array}$ & $\begin{array}{c}\text { Filles de 15- } \\
24 \text { ans }\end{array}$ \\
\hline Agadez & 31.2 & 37.8 & 34.5 \\
\hline Diffa & 13.1 & 10.6 & 12.7 \\
\hline Dosso & 40.3 & 37.9 & 39.7 \\
\hline Maradi & 48.7 & $(23.9)$ & 46.9 \\
\hline Tahoua & 32.5 & 30.5 & 32.2 \\
\hline Tillabéri & 48.2 & 36.2 & 45.4 \\
\hline Zinder & 14.3 & 8.3 & 13.4 \\
\hline Niamey & 19.4 & 16.1 & 17.4 \\
\hline Urbaine & $\mathbf{2 8 . 0}$ & $\mathbf{2 0 . 1}$ & $\mathbf{2 3 . 7}$ \\
\hline Rurale & $\mathbf{3 5 . 8}$ & $\mathbf{3 3 . 3}$ & $\mathbf{3 5 . 4}$ \\
\hline National & $\mathbf{3 4 . 7}$ & $\mathbf{2 6 . 1}$ & $\mathbf{3 2 . 8}$ \\
\hline
\end{tabular}

Lire la première colonne des données comme suit : « Le pourcentage des filles de 15-24 ans qui sont déjà mariées qui considèrent que la violence conjugale est acceptable quelles que soient les circonstances. »

Les chiffres entre parenthèses sont basés sur 25-50 cas non pondérés.

a Les filles ayant répondu par l'affirmative à toutes les cinq questions suivantes : Battre une femme est normal si 1) la femme peut sortir sans informer son mari 2) la femme néglige ses enfants 3) la femme argumente avec son mari 4) la femme refuse d'avoir des rapports sexuels avec son mari 5) la femme cuisine mal. 
Tableau 19B : Attitude des filles vis-à-vis de la violence conjugale

\begin{tabular}{|c|c|c|c|c|c|c|}
\hline \multicolumn{7}{|c|}{ Pourcentage des filles de 15-24 ans qui considèrent que la violence conjugale est } \\
acceptable dans certaines circonstances \\
\hline Région & $\begin{array}{c}\text { Si l'épouse est } \\
\text { sortie sans le } \\
\text { dire à son } \\
\text { mari }\end{array}$ & $\begin{array}{c}\text { Si } \\
\text { l'épouse } \\
\text { néglige } \\
\text { ses } \\
\text { enfants }\end{array}$ & $\begin{array}{c}\text { Si l'épouse } \\
\text { se dispute } \\
\text { avec son } \\
\text { mari }\end{array}$ & $\begin{array}{c}\text { Si } \\
\text { l'épouse } \\
\text { refuse } \\
\text { les } \\
\text { rapports } \\
\text { sexuels }\end{array}$ & $\begin{array}{c}\text { Si } \\
\text { l'épouse } \\
\text { cuisine } \\
\text { mal }\end{array}$ & $\begin{array}{c}\text { Filles ayant } \\
\text { répondu oui } \\
\text { à au moins } \\
\text { l'une de ces } \\
\text { questionsa }\end{array}$ \\
\hline Agadez & 59.1 & 58.9 & 48.0 & 52.5 & 42.5 & 69.9 \\
\hline Diffa & 28.1 & 26.3 & 33.6 & 41.9 & 25.3 & 49.9 \\
\hline Dosso & 75.8 & 70.6 & 66.1 & 73.2 & 53.6 & 89.1 \\
\hline Maradi & 60.1 & 57.8 & 54.5 & 60.7 & 55.9 & 71.1 \\
\hline Tahoua & 56.4 & 52.6 & 47.3 & 51.1 & 36.1 & 61.8 \\
\hline Tillabéri & 67.2 & 56.3 & 59.2 & 76.3 & 73.3 & 93.5 \\
\hline Zinder & 31.3 & 31.9 & 25.6 & 36.5 & 21.7 & 49.4 \\
\hline Niamey & 61.0 & 56.5 & 44.8 & 51.6 & 36.7 & 80.9 \\
\hline Urbain & $\mathbf{5 7 . 2}$ & $\mathbf{5 1 . 9}$ & $\mathbf{4 4 . 7}$ & $\mathbf{5 2 . 8}$ & $\mathbf{3 7 . 9}$ & $\mathbf{7 3 . 3}$ \\
\hline Rurale & $\mathbf{5 7 . 4}$ & $\mathbf{5 3 . 8}$ & $\mathbf{5 0 . 2}$ & $\mathbf{5 8 . 4}$ & $\mathbf{4 7 . 4}$ & $\mathbf{7 1 . 9}$ \\
\hline National & $\mathbf{5 7 . 3}$ & $\mathbf{5 3 . 4}$ & $\mathbf{4 9 . 0}$ & $\mathbf{5 7 . 2}$ & $\mathbf{4 5 . 2}$ & $\mathbf{7 2 . 2}$ \\
\hline
\end{tabular}

Lire la première colonne des données comme suit : « Le pourcentage des filles de 15-24 ans qui pensent que la violence conjugale est acceptable si elle sort sans informer son mari. »

a Filles ayant répondu oui à au moins l'une de ces cinq questions détaillées dans les colonnes précédentes. 


\section{Graphique 13 : Pourcentage des filles de 15-24 ans qui considèrent que la violence conjugale est acceptable dans certaines circonstances}

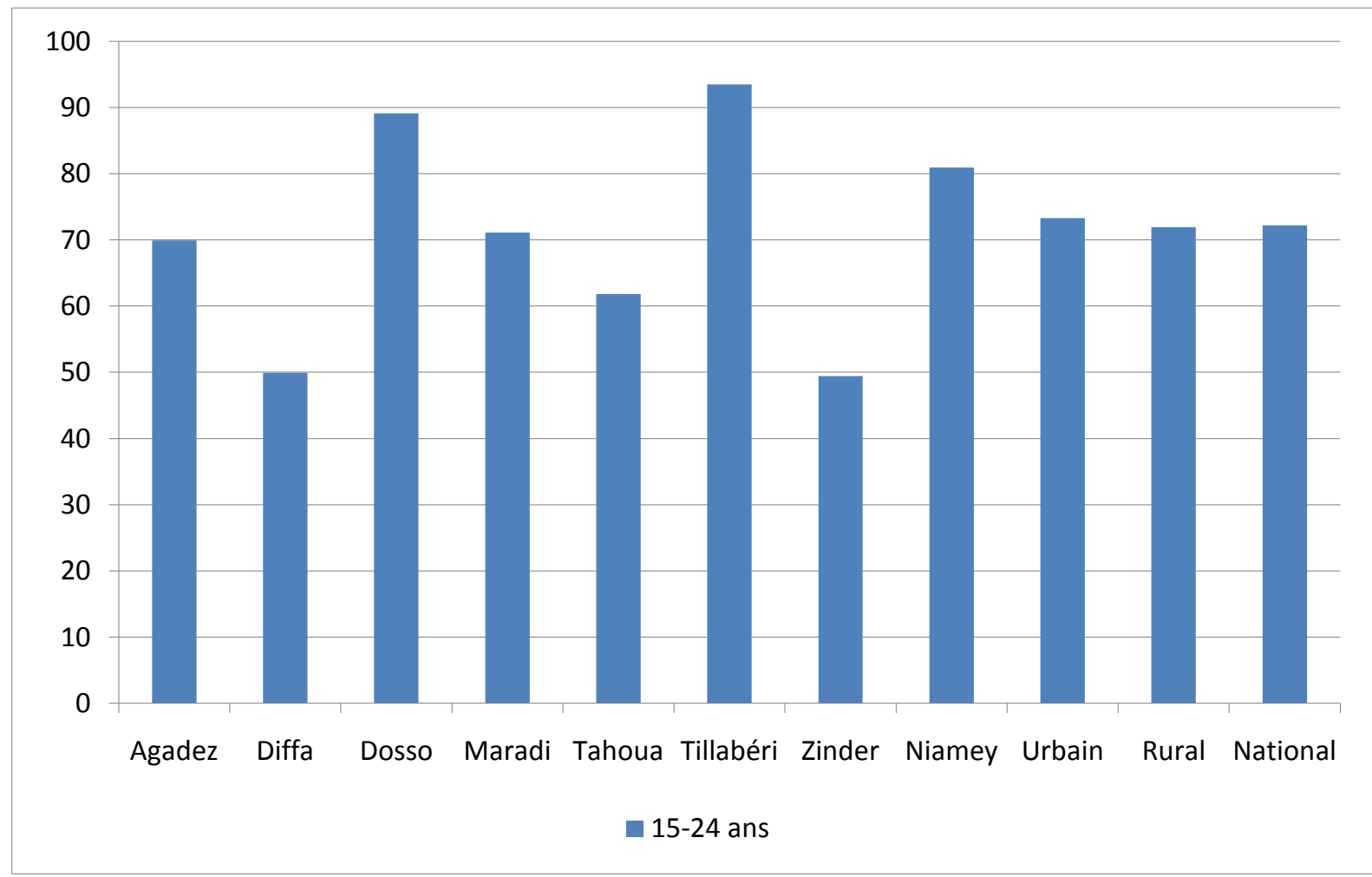

L'épidémie VIH touche de plus en plus de jeunes, et notamment de jeunes femmes Dans de nombreuses régions du globe, les infections par le VIH sont de plus en plus prévalentes chez les jeunes, et en particulier chez les jeunes femmes. Dans les pays d'Afrique subsaharienne, celles-ci sont le nouveau visage de la maladie. Le risque de contamination est d'autant plus élevé que ces jeunes femmes ou jeunes filles ont des ressources sociales et économiques limitées et qu'elles ne sont pas en position de gérer ou de refuser des relations sexuelles sans risque, que ce soit au sein du mariage ou en dehors ${ }^{10}$. Une analyse des Enquêtes Démographiques et de Santé (EDS) portant sur 31 pays permet de constater que la plupart des jeunes filles âgées de 15 à 19 ans qui sont sexuellement actives sont mariées ${ }^{11}$. Bien qu'il soit parfois présenté comment un moyen de protéger l'enfant, le mariage précoce est en réalité dangereux et pourrait même augmenter le risque d'infection par le VIH chez les jeunes filles. Celles-ci sont particulièrement vulnérables car on les pousse à avoir des enfants; la contraception $\mathrm{n}$ ' est donc pas pour elles une solution envisageable. Une communication franche entre partenaires sexuels permet de limiter les risques d'exposition au VIH, mais en raison des inégalités liées au genre dans de nombreux mariages et relations sexuelles, il est difficile

\footnotetext{
${ }^{10}$ Bruce, Judith. 2007. "Girls left behind: Redirecting HIV interventions toward the most vulnerable." Transitions to Adulthood Brief no. 23. New York: Population Council.

${ }^{11}$ Bruce, Judith and Shelley Clark. 2003. "Including Married Adolescents in Adolescent Reproductive Health and HIV/AIDS Policy," background paper prepared for the WHO/UNFPA/Population Council Technical Consultation on Married Adolescents, Geneva: 9-12 December
} 
pour les femmes - et en particulier pour les jeunes filles - de parler du VIH et de négocier pour que leur partenaire utilise un préservatif. Il est donc clair que bien qu'il soit indispensable que les jeunes soient informés sur le dépistage du VIH et sur les services qui leur sont offerts, cela ne suffit pas à les prémunir contre une infection. Des efforts importants doivent être entrepris pour atteindre les jeunes filles vulnérables et pour renforcer leur aptitude à se protéger.

Tableau 20 : Dépistage du VIH

\begin{tabular}{|c|c|c|c|c|c|c|}
\hline \multirow{2}{*}{ Région } & $\begin{array}{c}\text { Dilles ayant déjà fait } \\
\text { un dépistage du } \\
\text { VIH }\end{array}$ & $\begin{array}{c}\text { Filles ayant fait un } \\
\text { dépistage dans les } \\
12 \text { derniers mois }\end{array}$ & $\begin{array}{c}\text { Filles ayant fait un } \\
\text { dépistage ET } \\
\text { ayant reçu les } \\
\text { résultats }\end{array}$ \\
\cline { 2 - 7 } & $\begin{array}{c}|c| \\
15-19 \\
\text { ans }\end{array}$ & $\begin{array}{c}20-24 \\
\text { ans }\end{array}$ & $\begin{array}{c}15-19 \\
\text { ans }\end{array}$ & $\begin{array}{c}20-24 \\
\text { ans }\end{array}$ & $\begin{array}{c}15-19 \\
\text { ans }\end{array}$ & $\begin{array}{c}20-24 \\
\text { ans }\end{array}$ \\
\hline Agadez & 5.8 & 13.5 & 4.1 & 6.7 & 4.9 & 12.7 \\
\hline Diffa & 1.9 & 1.9 & 1.2 & 0.9 & 1.9 & 1.0 \\
\hline Dosso & 1.0 & 1.2 & 0.3 & 0.0 & 0.8 & 0.5 \\
\hline Maradi & 0.3 & 1.1 & 0.3 & 0.3 & 0.3 & 0.8 \\
\hline Tahoua & 1.5 & 2.5 & 0.8 & 1.1 & 1.3 & 2.3 \\
\hline Tillabéri & 0.5 & 0.5 & 0.0 & 0.5 & 0.4 & 0.5 \\
\hline Zinder & 2.0 & 1.7 & 2.0 & 0.6 & 2.0 & 1.4 \\
\hline Niamey & 7.6 & 11.4 & 3.2 & 6.4 & 6.6 & 10.3 \\
\hline Urbaine & $\mathbf{5 . 2}$ & $\mathbf{1 1 . 5}$ & $\mathbf{3 . 0}$ & $\mathbf{5 . 8}$ & $\mathbf{4 . 4}$ & $\mathbf{9 . 8}$ \\
\hline Rurale & $\mathbf{0 . 9}$ & $\mathbf{0 . 5}$ & $\mathbf{0 . 5}$ & $\mathbf{0 . 1}$ & $\mathbf{0 . 9}$ & $\mathbf{0 . 4}$ \\
\hline National & $\mathbf{2 . 0}$ & $\mathbf{2 . 9}$ & $\mathbf{1 . 1}$ & $\mathbf{1 . 3}$ & $\mathbf{1 . 7}$ & $\mathbf{2 . 5}$ \\
\hline
\end{tabular}

Lire la première colonne des données comme suit : « Le pourcentage des filles de 15-19 ans qui ont déjà fait un dépistage du VIH. » 
Graphique 14 : Pourcentage des filles de 15-24 ans qui ont fait un dépistage du VIH au cours des 12 derniers mois, par région

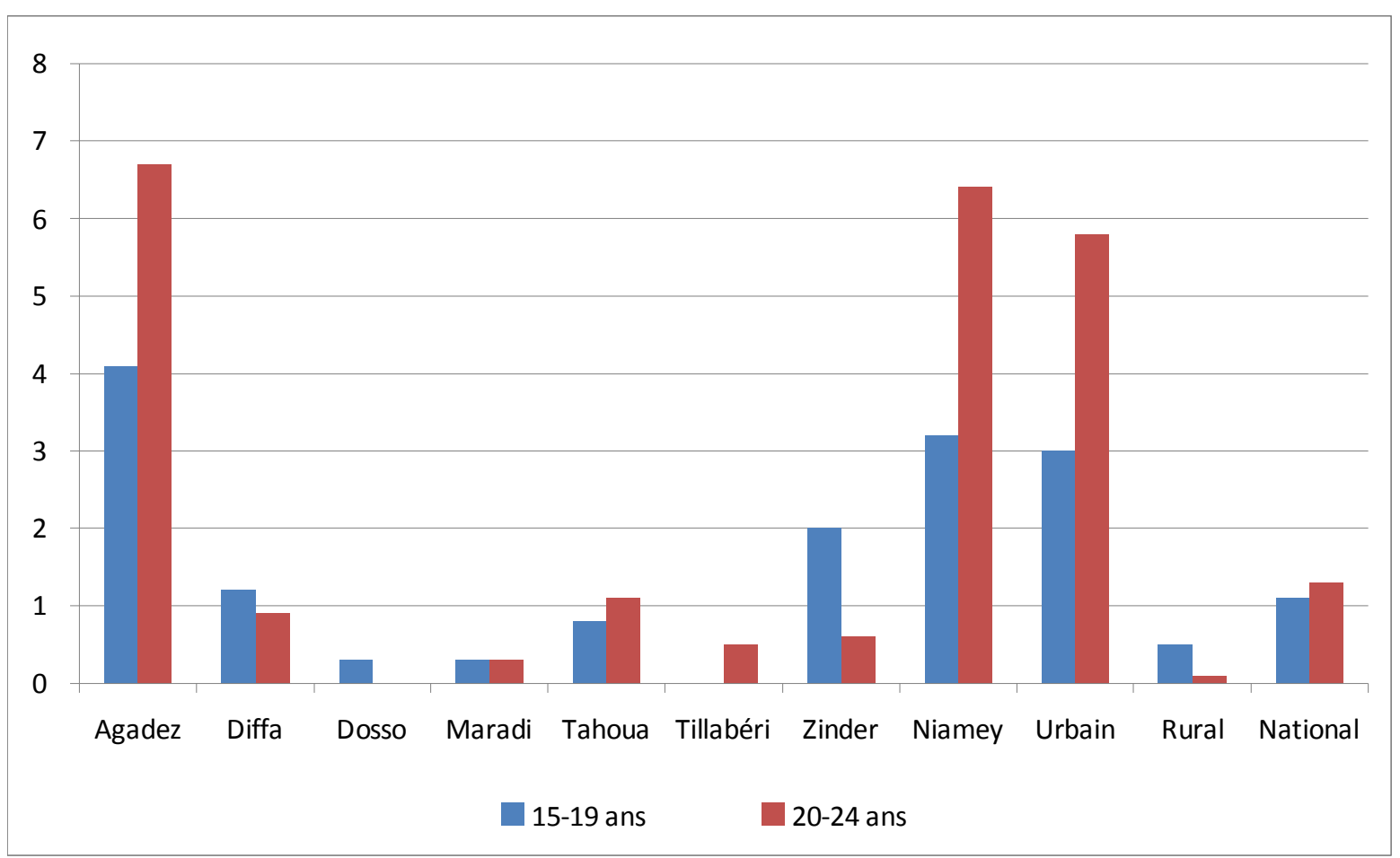

Tableau 21 : Connaissance des méthodes de prévention du VIH

\begin{tabular}{|c|c|c|c|}
\hline \multicolumn{3}{|c|}{$\begin{array}{c}\text { Connaissance des méthodes pour réduire le risque de contracter le sida } \\
\text { chez les filles de 15-24 ans (en \%) }\end{array}$} \\
\hline \multirow{2}{*}{ Région } & $\begin{array}{c}\text { N'ont des } \\
\text { Utilisent un } \\
\text { préservatif } \\
\text { rapports sexuels } \\
\text { qu'avec un seul } \\
\text { partenaire non } \\
\text { infecté }\end{array}$ & $\begin{array}{c}\text { N'ont pas de } \\
\text { rapports } \\
\text { sexuels }\end{array}$ \\
\hline Agadez & 62.6 & 81.1 & 71.2 \\
\hline Diffa & 64.0 & 92.1 & 71.4 \\
\hline Dosso & 70.4 & 94.9 & 90.0 \\
\hline Maradi & 81.6 & 86.9 & 78.8 \\
\hline Tahoua & 57.6 & 83.5 & 81.3 \\
\hline Tillabéri & 56.0 & 90.6 & 84.9 \\
\hline Zinder & 63.5 & 89.3 & 81.0 \\
\hline Niamey & 74.2 & 90.5 & 80.3 \\
\hline Urbaine & $\mathbf{7 5 . 8}$ & $\mathbf{8 9 . 8}$ & $\mathbf{8 1 . 3}$ \\
\hline Rurale & $\mathbf{6 2 . 9}$ & $\mathbf{8 8 . 5}$ & $\mathbf{8 2 . 5}$ \\
\hline National & $\mathbf{6 6 . 4}$ & $\mathbf{8 8 . 8}$ & $\mathbf{8 2 . 2}$ \\
\hline
\end{tabular}

Lire la première colonne des données comme suit : « Le pourcentage des filles de 15-24 ans qui savent qu'utiliser un préservatif est une méthode pour réduire le risque de contracter le sida. » 


\section{Le choix de la contraception et la sécurité contraceptive}

Pour assurer la santé sexuelle et de la procréation des adolescents, il faut leur permettre de faire le choix de la contraception et d'utiliser des contraceptifs sûrs. Les grossesses involontaires résultent d'un manque d'accès aux moyens de contraception modernes ou du fait que ceux-ci ne sont pas utilisés. La proportion de besoins non satisfaits en matière de contraception est bien plus élevée parmi les adolescents qui savent qu'il existe des moyens de contraception que dans le reste de la population. Une analyse des données des EDS menées dans 31 pays montre qu'en moyenne, $80 \%$ des rapports sexuels non protégés des jeunes filles ont lieu dans le cadre du mariage. En moyenne, dans les 25 pays pour lesquels ces données étaient disponibles , on a noté que $56,7 \%$ des jeunes filles mariées entre les âges de 15 et 19 ans avaient eu des rapports sexuels non protégés dans le courant de la semaine précédent l'enquête, alors que chez les jeunes filles sexuellement actives mais non mariées cette proportion était de $14,2 \% .12$ On ne sait pas exactement pourquoi plus de la moitié des jeunes filles mariées avait eu des relations sexuelles non protégées, mais on peut penser que ce phénomène est dû à des situations complexes et sensibles, allant du désir d'enfant à l'impossibilité de négocier des pratiques saines de santé de la procréation au sein de la relation. Qu'elles soient mariées ou non, les très jeunes filles et les jeunes filles sont certainement désavantagées du fait de leur âge et ne savent donc pas qu'elles peuvent négocier pour obtenir l'utilisation de contraceptifs ou n'ont pas l'autorité sociale pour négocier ; ceci est particulièrement vrai dans le cas d'une relation où le partenaire a davantage de sécurité économique et est fréquemment plus âgé. Comme elles ne sont souvent pas en mesure de négocier pour que soient utilisés des préservatifs masculins ou féminins, ces jeunes filles sont plus à risque d'attraper des MST, d'être infectées par le VIH, et d'avoir des grossesses non désirées. 
Tableau 22 : Attitude des filles vis-à-vis de l'utilisation du préservatif masculin

\begin{tabular}{|c|c|c|c|c|}
\hline \multicolumn{5}{|c|}{$\begin{array}{l}\text { Pourcentage des filles de 15-24 ans qui estiment qu'une femme } \\
\text { peut demander à son mari d'utiliser un préservatif s'il a une } \\
\text { MST }\end{array}$} \\
\hline \multirow[b]{2}{*}{ Région } & \multicolumn{2}{|c|}{ Ayant déjà été mariées } & \multicolumn{2}{|c|}{ Jamais mariées } \\
\hline & $15-19$ ans & $\begin{array}{c}20-24 \\
\text { ans }\end{array}$ & $15-19$ ans & $20-24$ ans \\
\hline Agadez & $(64.3)$ & 63.2 & 74.2 & $(66.8)$ \\
\hline Diffa & 71.5 & 70.7 & $(90.5)$ & $*$ \\
\hline Dosso & 71.0 & 74.1 & 74.1 & $*$ \\
\hline Maradi & 61.0 & 66.3 & $(78.6)$ & $*$ \\
\hline Tahoua & 84.0 & 80.2 & 68.9 & $*$ \\
\hline Tillabéri & 73.1 & 82.1 & 84.1 & $*$ \\
\hline Zinder & 55.2 & 56.5 & $(72.4)$ & * \\
\hline Niamey & $(84.9)$ & 85.0 & 83.9 & 92.8 \\
\hline Urbaine & 76.2 & 79.3 & 83.3 & 91.5 \\
\hline Rurale & 69.3 & 71.2 & 70.8 & (66.7) \\
\hline National & 70.1 & 72.5 & 77.4 & 87.2 \\
\hline
\end{tabular}

Lire la première colonne des données comme suit : « Le pourcentage des filles de 15-19 ans qui estiment qu'une femme peut demander à son mari d'utiliser un préservatif s'il a une MST. » Les chiffres entre parenthèses sont basés sur 25-50 cas non pondérés.

*Indique que le chiffre est basé sur moins de 25 cas non pondérés et a été supprimé.

\section{Tableau 23 : Utilisation du préservatif}

\begin{tabular}{|c|c|c|c|c|}
\hline \multicolumn{5}{|c|}{$\begin{array}{c}\text { Pourcentage des filles de 15-24 ans } \\
\text { lors du dernier rapport sexuel }\end{array}$} \\
\hline \multirow{2}{*}{ Région } & \multicolumn{2}{|c|}{$\begin{array}{c}\text { Actuellement mariées } \\
\text { ou en union }\end{array}$} & \multirow{2}{*}{$\begin{array}{c}\text { Jamais } \\
\text { mariées }\end{array}$} & $\begin{array}{c}15-24 \\
\text { ans }\end{array}$ \\
\cline { 2 - 3 } & $\begin{array}{c}\text { Avec } \\
\text { enfant }\end{array}$ & $\begin{array}{c}\text { Sans } \\
\text { enfant }\end{array}$ & & \\
\hline Urbaine & $\mathbf{1 . 7}$ & $\mathbf{2 . 5}$ & $\mathbf{( 2 2 . 5 )}$ & $\mathbf{2 . 7}$ \\
\hline Rurale & $\mathbf{0 . 2}$ & $\mathbf{0 . 2}$ & $*$ & $\mathbf{0 . 2}$ \\
\hline National & $\mathbf{0 . 4}$ & $\mathbf{0 . 4}$ & $\mathbf{( 1 9 . 4 )}$ & $\mathbf{0 . 6}$ \\
\hline
\end{tabular}

Lire la première colonne des données comme suit : « Le pourcentage des filles de 15-24 ans actuellement mariées ou en union avec au moins. » un enfant ayant utilisé un préservatif au dernier rapport sexuel. Les chiffres entre parenthèses sont basés sur 25-50 cas non pondérés.

* Indique que le chiffre est basé sur moins de 25 cas non pondérés et a été supprimé. aFilles de 15-24 ans qui rapportent d'avoir eu des rapports sexuels. 
Graphique 15: Pourcentage des filles de 15-24 ans ayant utilisé un préservatif lors du dernier rapport sexuel

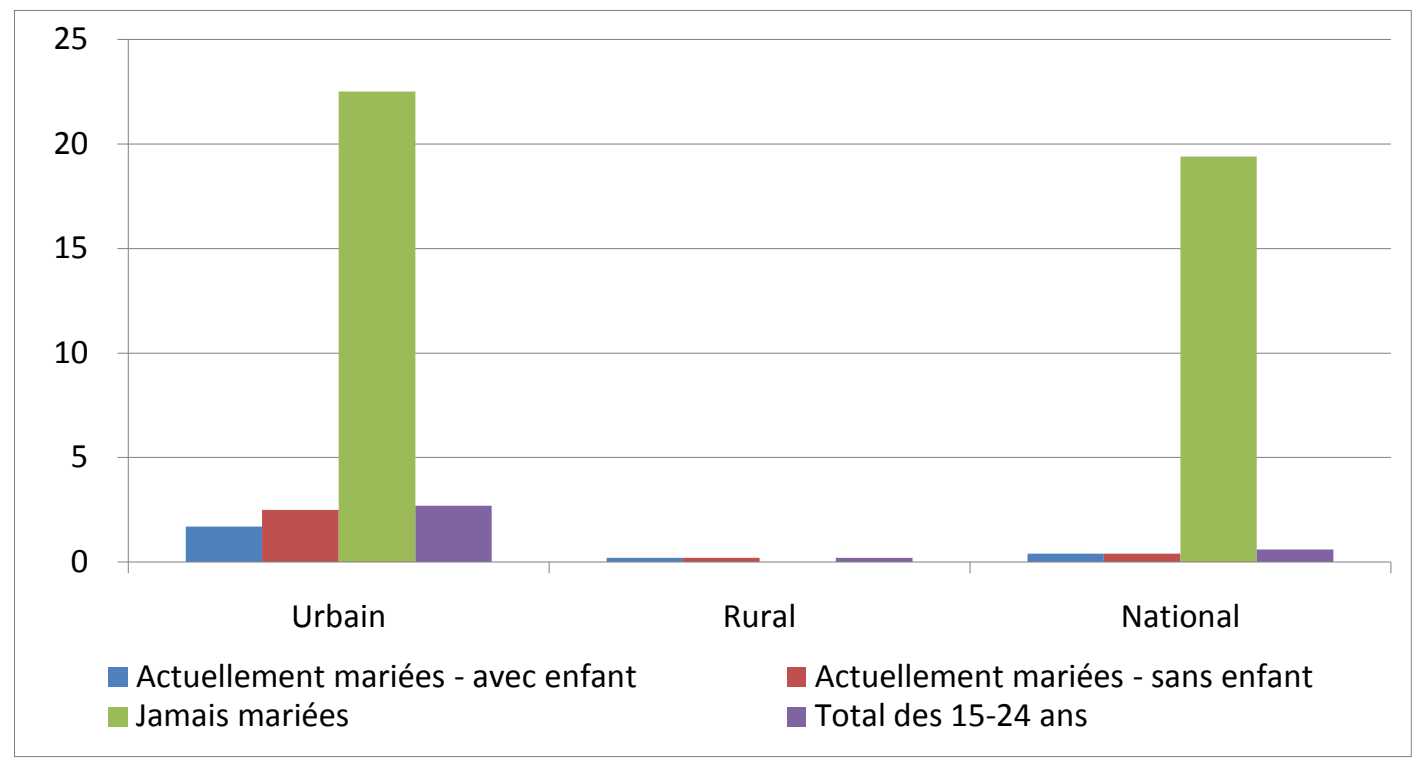


Tableau 24 : Utilisation de contraceptifs

\begin{tabular}{|c|c|c|c|c|}
\hline \multirow{2}{*}{$\begin{array}{c}\text { Pourcentage des filles de 14-24a ans ayant } \\
\text { déjà/n'ayant jamais utilisé de contraceptif }\end{array}$} \\
\hline \multirow{2}{*}{ Région } & \multicolumn{2}{|c|}{$\begin{array}{c}\text { Utilise un } \\
\text { contraceptif } \\
\text { moderne }\end{array}$} & $\begin{array}{c}\text { N'ayant jamais } \\
\text { utilisé de } \\
\text { contraceptif }\end{array}$ \\
\cline { 2 - 5 } & $\begin{array}{c}\text { Ayant } \\
\text { déjà été } \\
\text { mariées }\end{array}$ & $\begin{array}{c}\text { Jamais } \\
\text { mariées }\end{array}$ & $\begin{array}{c}\text { Ayant } \\
\text { déjà été } \\
\text { mariées }\end{array}$ & $\begin{array}{c}\text { Jamais } \\
\text { mariées }\end{array}$ \\
\hline Agadez & 10.9 & $*$ & 78.9 & $*$ \\
\hline Diffa & 5.7 & $*$ & 90.3 & $*$ \\
\hline Dosso & 13.2 & $*$ & 72.4 & $*$ \\
\hline Maradi & 1.9 & $*$ & 92.0 & $*$ \\
\hline Tahoua & 6.3 & $*$ & 88.8 & $*$ \\
\hline Tillabéri & 4.7 & $*$ & 77.7 & $*$ \\
\hline Zinder & 4.2 & $*$ & 88.2 & $*$ \\
\hline Niamey & 27.3 & $*$ & 65.8 & $*$ \\
\hline Urbaine & $\mathbf{2 4 . 8}$ & $\mathbf{( 1 8 . 1 )}$ & $\mathbf{6 7 . 3}$ & $\mathbf{( 6 4 . 9 )}$ \\
\hline Rurale & $\mathbf{4 . 1}$ & $*$ & $\mathbf{8 6 . 7}$ & $*$ \\
\hline $\begin{array}{c}\text { Niveau } \\
\text { national }\end{array}$ & $\mathbf{6 . 9}$ & $\mathbf{( 2 0 . 4 )}$ & $\mathbf{8 4 . 1}$ & $\mathbf{( 6 5 . 0 )}$ \\
\hline
\end{tabular}

Lire la première colonne des données comme suit : « Le pourcentage des filles de 15-24 ans mariées et qui ont déjà utilisé une méthode moderne de contraception. »

Les chiffres entre parenthèses sont basés sur 25-50 cas non pondérés.

* Indique que le chiffre est basé sur moins de 25 cas non pondérés et a été supprimé.

a Filles de 15-24 ans qui ont rapporté ayant eu des rapports sexuels.

\section{Soins prénataux et assistance à l'accouchement}

Dans les pays en développement, la grossesse et la maternité sont un défi pour les jeunes femmes. Il a été clairement établi que les fréquentes carences en matière de soins de santé - en particulier de santé maternelle - font courir de graves risques aux mères. Le risque de mort néonatale ou de mort du nourrisson, ainsi que le risque pour la mère, est beaucoup plus élevé chez les jeunes mères qui font une première grossesse, surtout si elles sont pauvres et peu éduquées. Le risque est particulièrement aigu quand la mère est très jeune et que son corps n'est pas encore assez développé pour supporter la grossesse. Pour obtenir des résultats satisfaisants dans le domaine de la santé maternelle et infantile, il est essentiel que les femmes aient accès à des services de santé maternelle adéquats. Ces services peuvent également jouer un rôle important et permettre d'identifier, voire de traiter, les MST et le VIH. Les décideurs politiques et les concepteurs de programmes doivent faire en sorte que la transition de ces jeunes filles vers la maternité se fasse dans de bonnes conditions de santé ; ceci implique la mise en place de services de santé de la procréation qui soient accessibles, adaptés, ciblés et taillés sur mesure pour répondre aux besoins spécifiques des jeunes filles qui sont mères pour la première fois. 
Tableau 25 : Santé prénatale

\begin{tabular}{|c|c|c|}
\hline \multicolumn{3}{|c|}{$\begin{array}{l}\text { Nombre moyen de consultations } \\
\text { prénatales }{ }^{\mathrm{a}} \text { chez les filles de 15-24 ans }\end{array}$} \\
\hline Région & $\begin{array}{c}15-19 \\
\text { ans }\end{array}$ & $\begin{array}{c}20-24 \\
\text { ans }\end{array}$ \\
\hline Agadez & $(2.3)$ & 2.5 \\
\hline Diffa & 1.3 & 1.6 \\
\hline Dosso & 1.5 & 1.8 \\
\hline Maradi & 1.4 & 1.1 \\
\hline Tahoua & 1.5 & 1.4 \\
\hline Tillabéri & 1.0 & 1.7 \\
\hline Zinder & 0.9 & 0.9 \\
\hline Niamey & $(2.8)$ & 3.0 \\
\hline Urbaine & $\mathbf{2 . 7}$ & $\mathbf{2 . 9}$ \\
\hline Rurale & $\mathbf{1 . 2}$ & $\mathbf{1 . 2}$ \\
\hline Niveau national & $\mathbf{1 . 3}$ & $\mathbf{1 . 5}$ \\
\hline
\end{tabular}

Lire la première colonne des données comme suit : « Le nombre de visites prénatales chez les filles de 15-19 ans. »

Les chiffres entre parenthèses sont basés sur 25-50 cas non pondérés.

a Pour la naissance la plus récente dans les cinq années précédant l'enquête. 
Tableau 26 : Assistance lors de l'accouchement

\begin{tabular}{|c|c|c|c|c|c|c|c|c|c|c|}
\hline \multicolumn{11}{|c|}{$\begin{array}{c}\text { Pourcentage des filles âgées } 15 \text { à } 24 \text { ans ayant reçu une assistance }{ }^{a} \text { lors de l'accouchement, quelle } \\
\text { que soit sa forme }\end{array}$} \\
\hline \multirow{2}{*}{ Région } & \multicolumn{2}{|c|}{$\begin{array}{c}\text { Professionnel de } \\
\text { santé }\end{array}$} & \multicolumn{2}{|c|}{$\begin{array}{c}\text { Sage-femme } \\
\text { avec formation }\end{array}$} & \multicolumn{2}{|c|}{$\begin{array}{l}\text { Sage-femme } \\
\text { traditionnelle }\end{array}$} & \multicolumn{2}{|c|}{ Autre ${ }^{b}$} & \multicolumn{2}{|c|}{$\begin{array}{c}\text { Aucune } \\
\text { assistance }\end{array}$} \\
\hline & $\begin{array}{c}15-19 \\
\text { ans }\end{array}$ & $\begin{array}{c}20-24 \\
\text { ans }\end{array}$ & $\begin{array}{c}15-19 \\
\text { ans }\end{array}$ & $\begin{array}{c}20-24 \\
\text { ans }\end{array}$ & $\begin{array}{c}15-19 \\
\text { ans }\end{array}$ & $\begin{array}{c}20-24 \\
\text { ans }\end{array}$ & $\begin{array}{c}15-19 \\
\text { ans }\end{array}$ & $\begin{array}{c}20-24 \\
\text { ans }\end{array}$ & $\begin{array}{c}15-19 \\
\text { ans }\end{array}$ & $\begin{array}{c}20-24 \\
\text { ans }\end{array}$ \\
\hline Agadez & $(63.2)$ & 41.4 & $(4.4)$ & 15.8 & $(30.2)$ & 33.6 & $(0.0)$ & 0.0 & $(2.2)$ & 9.3 \\
\hline Diffa & 14.5 & 13.6 & 51.3 & 39.2 & 33.1 & 39.6 & 0.0 & 0.0 & 1.2 & 7.6 \\
\hline Dosso & 18.1 & 20.7 & 12.1 & 9.0 & 36.4 & 30.2 & 0.0 & 0.5 & 33.4 & 39.8 \\
\hline Maradi & 14.7 & 15.1 & 6.5 & 12.1 & 63.7 & 59.4 & 0.0 & 0.0 & 15.1 & 13.5 \\
\hline Tahoua & 20.6 & 20.3 & 19.0 & 16.6 & 55.0 & 53.6 & 1.5 & 0.0 & 3.9 & 9.6 \\
\hline Tillabéri & 14.8 & 17.2 & 14.4 & 20.1 & 61.5 & 49.1 & 0.0 & 0.0 & 9.3 & 13.7 \\
\hline Zinder & 14.2 & 11.2 & 10.4 & 12.4 & 70.6 & 54.8 & 0.0 & 0.3 & 4.8 & 21.4 \\
\hline Niamey & $(76.5)$ & 72.9 & $(11.0)$ & 9.1 & $(9.2)$ & 8.9 & $(0.0)$ & 0.5 & $(3.3)$ & 8.7 \\
\hline Urbaine & 71.7 & 74.5 & 6.4 & 6.7 & 17.7 & 10.0 & 0.0 & 0.2 & 4.2 & 8.6 \\
\hline Rurale & 12.1 & 9.8 & 14.5 & 15.7 & 60.7 & 55.0 & 0.4 & 0.1 & 12.3 & 19.4 \\
\hline $\begin{array}{l}\text { Niveau } \\
\text { national }\end{array}$ & 19.6 & 20.8 & 13.5 & 14.1 & 55.3 & 47.4 & 0.3 & 0.2 & 11.3 & 17.6 \\
\hline
\end{tabular}

Lire la première colonne des données comme suit: «Le pourcentage des femmes de 15-19 ans qui ont reçu une assistance d'un professionnel de santé lors de l'accouchement. »

Si le répondent a mentionné l'assistance de plus d'un personnel de santé lors de l'accouchement, seulement la personne la plus qualifiée est considérée dans ce tableau.

Les chiffres entre parenthèses sont basés sur 25-50 cas non pondérés.

a Pour la naissance la plus récente dans les cinq années précédant l'enquête.

${ }^{b}$ D'autres types inclut les amies ou la famille. 
Graphique 16 : Filles de 15-24 ans ayant reçu une assistance lors de l'accouchement

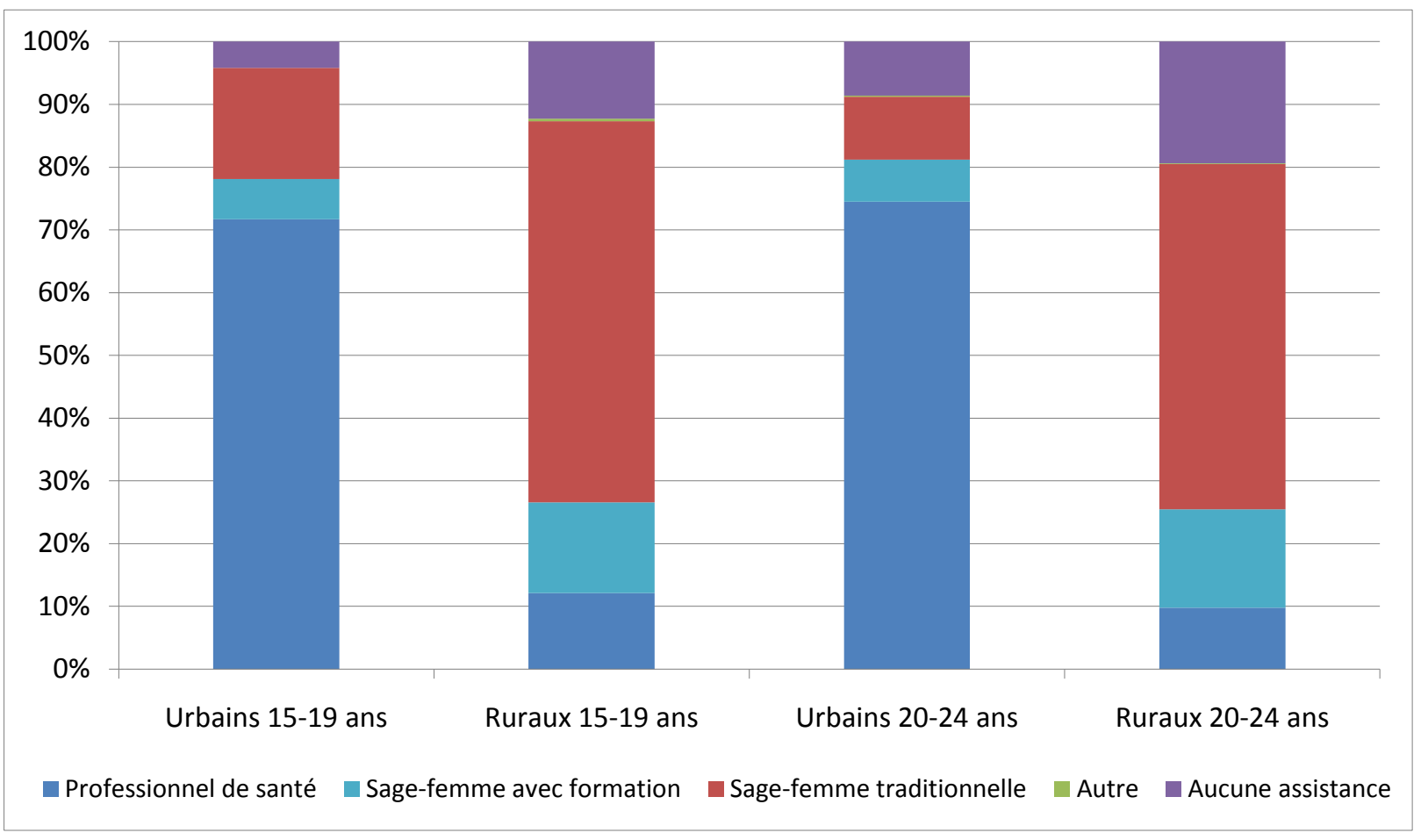




\section{$\underline{V}$-Quelques remarques pour conclure}

Les données contenues dans ce rapport constituent l'un des éléments essentiels à l'élaboration d'une stratégie documentée destinée à mettre en place des politiques et des programmes ciblés permettant d'aider les jeunes les plus vulnérables. Comme les indicateurs sélectionnés couvrent une vaste gamme de contextes et de scenarii, les décideurs politiques et les personnes chargées de concevoir des programmes, quel que soit leur secteur, peuvent commencer à plaider en faveur d'investissements ciblés destinés à aider les plus vulnérables, notamment les jeunes filles. Cependant, du fait des limites des EDS, les données ne sont pas exhaustives et ne couvrent pas tous les domaines nécessaires à la mise en place de plaidoyers au niveau local. Les équipes sur le terrain ont certainement accès à d'autres sources de données qualitatives et quantitatives qui permettront de combler les lacunes en fonction des besoins de ces acteurs locaux. De ce fait il serait très utile à notre effort que les utilisateurs de ce guide de données commencent à conceptualiser le tableau dressé en incluant des remarques spécifiques pour chaque pays, qui se fondent sur leur expérience et l'observation de programmes, lorsqu'ils intégreront de nouvelles sources de données. Ceci renforcera l'efficacité d'ensemble de ce guide de données en permettant aux agences locales des Nations Unies et aux structures gouvernementales et non gouvernementales de mieux comprendre que les priorités nationales et les objectifs à moyen terme ont un lien direct avec les données régionales qui déterminent l'analyse des politiques, la conception des programmes, et les efforts de renforcement des capacités. 